\title{
Nanoscale hydrodynamics near solids
}

Cite as: J. Chem. Phys. 148, 064107 (2018); https://doi.org/10.1063/1.5010401

Submitted: 25 October 2017 . Accepted: 24 January 2018 . Published Online: 12 February 2018

Diego Camargo (D), J. A. de la Torre (D), D. Duque-Zumajo, Pep Español (D), Rafael Delgado-Buscalioni (iD), and Farid Chejne

\section{ARTICLES YOU MAY BE INTERESTED IN}

Perspective: Dissipative particle dynamics

The Journal of Chemical Physics 146, 150901 (2017); https://doi.org/10.1063/1.4979514

Hydrodynamic relaxations in dissipative particle dynamics

The Journal of Chemical Physics 148, 034503 (2018); https://doi.org/10.1063/1.4986569

Calculation of a solid/liquid surface tension: A methodological study

The Journal of Chemical Physics 148, 034702 (2018); https://doi.org/10.1063/1.5008473

\section{Where in the world is AIP Publishing?}

Find out where we are exhibiting next 


\title{
Nanoscale hydrodynamics near solids
}

\author{
Diego Camargo, ${ }^{1,2}$ J. A. de la Torre, ${ }^{3}$ D. Duque-Zumajo, ${ }^{3}$ Pep Español, ${ }^{3}$ \\ Rafael Delgado-Buscalioni, ${ }^{4}$ and Farid Chejne ${ }^{1}$ \\ ${ }^{1}$ Facultad de Minas, Universidad Nacional de Colombia, Medellin, Colombia \\ ${ }^{2}$ Facultad Mecánica, Universidad Pontificia Bolivariana, Montería, Colombia \\ ${ }^{3}$ Departamento Física Fundamental, Universidad Nacional de Educación a Distancia, \\ Aptdo. 60141, E-28080 Madrid, Spain \\ ${ }^{4}$ Departamento de Física Teórica de la Materia Condensada, Universidad Autónoma de Madrid, \\ Madrid 28049, Spain
}

(Received 25 October 2017; accepted 24 January 2018; published online 12 February 2018)

\begin{abstract}
Density Functional Theory (DFT) is a successful and well-established theory for the study of the structure of simple and complex fluids at equilibrium. The theory has been generalized to dynamical situations when the underlying dynamics is diffusive as in, for example, colloidal systems. However, there is no such a clear foundation for Dynamic DFT (DDFT) for the case of simple fluids in contact with solid walls. In this work, we derive DDFT for simple fluids by including not only the mass density field but also the momentum density field of the fluid. The standard projection operator method based on the Kawasaki-Gunton operator is used for deriving the equations for the average value of these fields. The solid is described as featureless under the assumption that all the internal degrees of freedom of the solid relax much faster than those of the fluid (solid elasticity is irrelevant). The fluid moves according to a set of non-local hydrodynamic equations that include explicitly the forces due to the solid. These forces are of two types, reversible forces emerging from the free energy density functional, and accounting for impenetrability of the solid, and irreversible forces that involve the velocity of both the fluid and the solid. These forces are localized in the vicinity of the solid surface. The resulting hydrodynamic equations should allow one to study dynamical regimes of simple fluids in contact with solid objects in isothermal situations. Published by AIP Publishing. https://doi.org/10.1063/1.5010401
\end{abstract}

\section{INTRODUCTION}

There is great interest in the behaviour of fluids in the nanoscale ${ }^{1}$ and microscale., ${ }^{2,3}$ At nanoscales, the structure of the fluid starts playing an important role. The standard description of structured fluids is based on the (classic) Density Functional Theory (DFT) ${ }^{4}$ with the density functional capturing all the relevant information about the equilibrium state of the fluid. In recent years, there has been great interest in obtaining dynamic versions of the Density Functional Theory (DDFT) that would allow one to discuss not only equilibrium structured fluids and its correlations but also their dynamic behaviour. ${ }^{5,6}$ Since the pioneering work by Marconi and Tarazona, ${ }^{7}$ which was based on a Smoluchowski description for colloidal particles, several approaches have been considered for the formulation of dynamic versions of DFT, ranging from kinetic theory ${ }^{8}$ to projection operators ${ }^{9}$ and variational approaches. ${ }^{10}$ Most existing studies deal with colloidal suspensions. ${ }^{6,11}$ However, there still exists a gap in the treatment of the dynamics of simple fluids near solids at scales where the structure of the fluid is relevant. Note that the interaction of the solid with the fluid is responsible for the structuring of the density field near a wall (which is a purely reversible and equilibrium effect) but also for irreversible processes that can be understood, at macroscales, as boundary conditions for the fluid. ${ }^{12,13}$
In Ref. 9, we have formulated DDFT based on projection operator techniques, where the density field is the only relevant variable. This is appropriate for colloidal systems that are suspended in a solvent acting as a thermal bath. In that case, the density field should be rather understood as the concentration field which, being conserved, is expected to be a slow variable. By selecting the density field as the only relevant variable, we are implicitly assuming that the density evolves in time scales which are much larger than the time scales corresponding to other variables in the system. In contrast to colloidal systems, simple fluids have the momentum density and energy density as conserved variables, and these variables may evolve in comparable time scales as the density. We have recently extended DDFT to non-isothermal situations by including the energy density field. ${ }^{14}$ Recent attempts in that direction have also been taken by Schmidt ${ }^{15}$ and Wittkowski et al. ${ }^{16}$ The resulting theory is valid for quiescent fluids or solids in which convective motion is not present. In the present work, we consider the mass and momentum density fields of a fluid, allowing us to address moving fluids, but we do not consider energy transfer. That is, we assume that momentum transfer is not affected by energy fluxes. This is the case in isothermal processes or even under slight temperature differences whenever the adiabatic coefficient is close to one, as corresponds to a practically incompressible liquid like water at room temperature. The natural thermodynamic potential is 
the free energy functional instead of an entropy functional as introduced in Ref. 14. In future work, we will address the energy transport at nanoscales.

In addition, we consider that the fluid is moving in the presence of a large spherical solid particle in order to address fluid-solid interactions. In usual DFT approaches, a solid wall is usually represented with an external hard potential. In the present description, a solid wall is treated with a coarse-grained (CG) procedure in which the atoms of the wall are eliminated from the description, under the assumption that any elastic (or any other) degree of freedom of the solid is much faster than the time scales of the surrounding fluid. The density functional that emerges now depends not only on the density field of the fluid but also on the overall CG variables that we use to describe the solid which, in the present work, are just its position and momentum. For simplicity, we focus on a particularly simple particle shape, a solid spherical particle. By considering the interaction of a fluid with a solid sphere, we may address the issue of total momentum conservation that may become rather obscure if one considers "planar walls with infinite mass." Of course, many of the results that we obtain will be easily transferred to planar walls, as limits in which the mass and the radius of the solid sphere are both very large. In addition, we take a spherical particle because only then the position and momentum of the center of mass of the solid particle is required in order to have a coarse-grained description of the solid. For non-spherical particles, it is necessary, in general, to include also the orientation and angular velocity of the particle, as this is expected to play an important role in the dynamics. Still, even in the spherical particle case, it may be important to introduce angular velocity in order to have accurate results. However, for the sake of simplicity and presentation of the basic results, we restrict ourselves in this paper to the simplest case without angular variables for the solid particle. Also, and for the sake of simplicity, we do not consider the intrinsic spin of the fluid that may become an important relevant variable at nanoscales. ${ }^{17,18}$

The main result of the present work is a set of dynamic equations that govern the non-equilibrium averages of hydrodynamic variables and the time-dependent position and momentum of the sphere. The dynamic equations are of the form of non-local hydrodynamic equations for the mass and momentum density fields coupled with Newton's laws for the center of mass of the solid particle. The coarse-grained forces between the fluid and the solid have reversible and dissipative contributions, both localized in a boundary region near the solid surface. The hydrodynamic equations obtained can describe the structuring of the fluid near the solid particle and non-local flow effects that may be important at nanoscales.

The present theory is both (i) a generalization of density functional theory to dynamic situations in simple fluids (fluids that obey a Hamiltonian dynamics) and (ii) a full description, at the coarse-grained hydrodynamic level, of the solid-wall interactions. The theory describes hydrodynamics at scales where the molecular structure of the fluid is apparent. At these scales, the concept of boundary condition is not applicable. Instead, the interaction of the fluid with the solid wall is described with reversible and irreversible forces confined at the vicinity of the wall.
Because of the two aspects of the present theory that have been already mentioned, i.e., (i) non-local hydrodynamics and (ii) interactions with solid walls, we discuss previous work in these two areas in what follows.

\section{A. Non-local hydrodynamics}

Our work relies on the pioneering work by Piccirelli ${ }^{19}$ who, following Robertson, ${ }^{20}$ derived hydrodynamic equations explicitly from the microscopic dynamics of the fluid system (see also the textbook by Grabert ${ }^{21}$ ). The resulting exact hydrodynamic equations contained non-local transport coefficients defined in terms of Green-Kubo formulae. In similar terms, Grossmann ${ }^{22}$ presented a derivation of non-local hydrodynamics of simple fluids by using essentially the same ideas behind the projection operator technique. While the theory provided non-local transport coefficients, no connection was made in these early studies with density functional theory, which was not yet developed in those days. Such non-local versions for hydrodynamics are necessary when the flow fields vary rapidly in space, in the range of molecular correlations. The probing of nanoscales in molecular dynamics (MD) simulations has demonstrated this point very convincingly. ${ }^{23-26}$

\section{B. Fluid-solid interactions}

Usually, at the hydrodynamic level of description, the interaction of a fluid with a solid is described through boundary conditions. Seminal work on the understanding of boundary conditions as irreversible interfacial processes was given by Bedeaux, Albano, and Mazur, who introduced interfacial transport coefficients entering into the boundary conditions. ${ }^{12}$ While a fluctuation-dissipation theorem was formulated for the slip coefficient, ${ }^{27}$ which could suggest a microscopic evaluation of the interfacial boundary coefficients in terms of molecular dynamics, it was not until the contribution by Bocquet and Barrat that microscopic expressions for the interfacial mechanical ${ }^{13}$ and thermal ${ }^{28}$ slip transport coefficients were given. This work was a conceptual breakthrough in the extensive field of flow slip at solid surfaces. However, a debate was initiated by Petravic and Harrowell ${ }^{29}$ who pointed out that the Green-Kubo expression proposed by Bocquet and Barrat provides not an intrinsic solid-fluid property but rather it depends on the geometry of the setup. This is due to the fact that the original expression reflects the total fluid friction for shear flow, including the slip friction at both interfaces as well as the viscous friction in the fluid. ${ }^{26,30}$ Hansen et al. ${ }^{26}$ propose to look at a thin slab of fluid near the wall and propose a phenomenological Langevin equation relating the velocity of the center of mass of the fluid slab and the force that the wall exerts on the slab. The friction coefficient may be computed from equilibrium MD simulations by comparing the velocityforce correlation to the velocity-velocity correlations of the slab. Another recent approach has considered a generalized Langevin equation for the velocity of a single atom of the fluid near a wall. ${ }^{31}$ Recent work by Ramos-Alvarado et al. ${ }^{32}$ compares the above different approaches and concludes that simulations are very sensitive to post-processing issues, leaving the story somewhat inconclusive.

Our theoretical approach is not restricted to parallel flow (i.e., fluid slabs) as is typically considered in simulation 
work. ${ }^{13,26,29,30,32}$ Most derivations of Green-Kubo expressions for friction are based on linear response theory where the Hamiltonian is slightly perturbed by an external forcing. ${ }^{13,31}$ We follow here a different approach in obtaining directly the full hydrodynamic equations from the microscopic dynamics. In this way, the transport coefficients that we obtain are the ones that really enter the equations of hydrodynamics in any general flow configuration, not limited to homogeneous isotropic flat walls.

Note that all the mentioned work on MD simulations is directed to compute the transport coefficients that appear in slip boundary conditions. When one descends to the nanoscale, however, the picture of the effect of the solid-fluid interactions in terms of a boundary condition on an idealized surface is questionable. For the scales in which nanostructure is visible, e.g., layering near the wall, we aim at describing the fluid solid interaction through coarse-grained forces that extend in a short range from the solid. An early attempt to use a "friction field" appeared in Ref. 33. Only when the fluid is macroscopic, we would expect these forces to be treated as singular forces such that its effect can be described as truly boundary conditions on the fluid. In a future publication, we will describe how the present theory gives rise to boundary conditions when the flows are macroscopic.

A precursor of our work is Ref. 34 that considered a Brownian hard sphere in a sea of small hard spheres and used both Mori projection operator and kinetic theory to derive hydrodynamic equations coupled to the motion of the Brownian particle (without boundary conditions). Our method is more general in that continuum pair-wise potentials are allowed for, and the non-linear Kawasaki-Gunton projection operator ${ }^{35}$ is used for the coarse-graining procedure instead of the simpler Mori projector, ${ }^{31}$ the latter being limited to near equilibrium linear equations of motion. ${ }^{21} \mathrm{~A}$ theory of coarse-graining based on the Kawasaki-Gunton projector gives a universal structure based on the usual thermodynamic potentials. The use of the Kawasaki-Gunton projector allows us to express the reversible part of the dynamics in a way that generalizes density functional theory to moving fluids.

Finally, Cadusch et al. ${ }^{36}$ show that the use of a non-local translation invariant kernel is not exempt of problems in high density fluids in strong nanoconfinement. They state "the fundamental theoretical challenge that remains is to include the position dependence into the kernel so that it becomes a genuinely inhomogeneous function of space and also to appropriately model the boundary conditions at the fluid-wall interface, including stick/slip boundary conditions." The present work addresses this challenge.

\section{NON-EQUILIBRIUM STATISTICAL MECHANICS}

We review in this section the well-established theory of non-equilibrium statistical mechanics. ${ }^{21}$ As in equilibrium, the theory is based on the notion of microstates and macrostates. Consider a set of phase functions $\hat{A}(z)$ where $z$ is the microscopic state of the system, i.e., the collection of positions and momenta of every atom in the system. These sets of variables are the selected coarse-grained (CG) variables and this selection determines the level of description at which the system is being monitored. In general, $\hat{A}(z)$ includes the total energy of the system. The impossibility of knowing the initial conditions for $z$ in a macroscopic system enforces the introduction of a probability density that describes in a statistical way the system. The average of the CG function $\hat{A}(z)$ with respect to the probability density is denoted with

$$
a=\operatorname{Tr}[\hat{A} \rho],
$$

where the classical trace operation $\operatorname{Tr}[\cdots]$ denotes an integration over $z$ (and a sum over particle number in a grand canonical setting). Along this paper, phase functions are denoted with a hat, while their averages will be unhatted.

\section{A. The entropy}

As well as in equilibrium, in non-equilibrium situations entropy plays a fundamental role. We define in this section the entropy of a given level of description. In the space of probability densities, the (dimensionless) Gibbs-Jaynes entropy functional is defined as

$$
\mathcal{S}[\rho]=-\operatorname{Tr}\left[\rho \ln \frac{\rho}{\rho_{0}}\right],
$$

where $\rho_{0}=\frac{1}{N ! h^{3 N}}$, with $h$ being the Planck's constant, is a dimensional factor that renders the argument of the logarithm dimensionless and that takes into account the proper Boltzmann counting. The trace symbol denotes a macrocanonical sum over particles and an integral over the position and momentum of $N$ particles. The normalized probability density that maximizes the entropy functional, subject to produce prescribed values of the averages (1), is denoted as the relevant ensemble $\bar{\rho}$ and has the form of a generalized canonical ensemble

$$
\bar{\rho}(z)=\frac{1}{Z[\lambda]} \rho_{0} \exp \{-\lambda \cdot \hat{A}(z)\},
$$

where $\lambda$ is the set of variables conjugate to the relevant variables $\hat{A}(z)$. The generalized partition function is given by

$$
Z[\lambda]=\operatorname{Tr}\left[\rho_{0} \exp \{-\lambda \cdot \hat{A}\}\right] .
$$

In general, $\lambda$ will be a collection of fields and finite dimensional vectors. We use the notation $[\cdots]$, which is typically restricted to denote a functional, also in the present mixed case. The average $a$ of the relevant variables with respect to the relevant ensemble will be denoted by

$$
a=\langle\hat{A}\rangle^{\lambda}=\operatorname{Tr}[\bar{\rho} \hat{A}]
$$

and can be written as

$$
a=\frac{\partial \Phi}{\partial \lambda}[\lambda],
$$

where the (dimensionless) thermodynamic potential $\Phi[\lambda]$ is given by

$$
\Phi[\lambda]=-\ln Z[\lambda] .
$$

The average $a$ is a function/functional of $\lambda$. For each $\lambda$, we have an average $a$ given by Eq. (6). If we take the derivative of (6) with respect to $\lambda$, we arrive at

$$
\frac{\partial a}{\partial \lambda}=-\langle\delta \hat{A} \delta \hat{A}\rangle^{\lambda}
$$

where $\delta A=\hat{A}(z)-a$. The covariance $\langle\delta \hat{A} \delta \hat{A}\rangle^{\lambda}$ is a positive definite matrix and, therefore, the functional $\Phi[\lambda]$ is convex. 
This implies that the Jacobian of the change of variables from $\lambda$ to $a$ can be inverted to provide $\lambda[a]$. Therefore, there is a one to one connection between the pair of conjugate variables $\lambda$ and $a$. This argument is valid for any pair of conjugate variables and it only depends on the definition of the conjugate variables introduced in Eq. (3). It constitutes the basic content of the DFT when the relevant variable is the microscopic density operator.

Because the connection is one to one, we may change variables from $\lambda$ to $a$. However, the average $a$ is given by a derivative and such a change of variables implies a loss of information. As we know from the usual treatment in thermodynamics, ${ }^{37}$ the correct way to proceed is to introduce the dimensionless entropy function $S[a]$ of the given level of description as the (minus) Legendre transform of the thermodynamic potential in the form

$$
S[a]=-\Phi[\lambda[a]]+\lambda[a] a .
$$

The relation of this entropy function $S[a]$ and the Gibbs-Jaynes entropy functional $\mathcal{S}[\bar{\rho}]$ in (2) is simple. The former is just the later evaluated at its maximum, the relevant ensemble (3). That is,

$$
S[a]=\mathcal{S}[\bar{\rho}] .
$$

Because the entropy $S[a]$ is the Legendre transform of the thermodynamic potential $\Phi[\lambda]$, we have the relationship conjugate to $(6)$,

$$
\frac{\partial S}{\partial a}=\lambda
$$

\section{B. The dynamics}

In general, it is possible to derive the evolution equation for a given dynamic variable by using the technique of projection operators. ${ }^{21,35}$ The projection operator method can be understood, at its most fundamental level as a way to approximate the actual time-dependent ensemble, which is the solution of the Liouville equation, with a relevant ensemble of the form (3), plus a correction, which is responsible for the irreversible behaviour. We summarize in the rest of this section the timedependent projection operator technique as presented in the classical textbook by Grabert. ${ }^{21}$

The aim is to derive equations of motion for the timedependent average $a_{i}(t)$ of the set of relevant variables $\hat{A}_{i}(z)$. The time-dependent average is

$$
a_{i}(t)=\operatorname{Tr}\left[\rho_{t} \hat{A}_{i}\right],
$$

where $\rho_{t}$ is the non-equilibrium solution of the Liouville equation. As it is shown in Ref. 21, for isolated systems with a timeindependent Hamiltonian, the averages (12) evolve according to the following closed exact equation:

$$
\frac{\partial}{\partial t} a_{i}(t)=v_{i}(t)+\int_{0}^{t} d t^{\prime} \sum_{j} K_{i j}\left(t, t^{\prime}\right) \lambda_{j}\left(t^{\prime}\right) .
$$

The reversible term is given by

$$
v_{i}(t)=\operatorname{Tr}\left[\bar{\rho}_{t} i L \hat{A}_{i}\right],
$$

where the Liouville operator is

$$
i L=-\sum_{i}\left[\frac{\partial H}{\partial \mathbf{q}_{i}} \frac{\partial}{\partial \mathbf{p}_{i}}-\frac{\partial H}{\partial \mathbf{p}_{i}} \frac{\partial}{\partial \mathbf{q}_{i}}\right]
$$

and $H(z)$ is the Hamiltonian of the system. The relevant ensemble $\bar{\rho}_{t}$ is of the form (3), with a time-dependent conjugate variable $\lambda(t)$. The conjugate variables $\lambda$ are selected in such a way that the averages $a(t)$ of the real and of the relevant ensemble coincide. Note that if only the reversible term $v_{i}(t)$ would be present in Eq. (13), we would be approximating the actual ensemble that it is a solution of the Liouville equation with a relevant ensemble of the form (3) where the conjugate field $\lambda(t)$ is now a function of time. The error in this approximation is, in fact, the memory term that describes irreversible behaviour. The irreversible term in Eq. (13) involves the memory kernel

$$
K_{i j}\left(t, t^{\prime}\right)=\operatorname{Tr}\left[\bar{\rho}_{t^{\prime}}\left(\mathcal{Q}_{t^{\prime}} i L \hat{A}_{j}\right) G_{t^{\prime} t}\left(\mathcal{Q}_{t} i L \hat{A}_{i}\right)\right],
$$

where the Kawasaki-Gunton projection operator $\mathcal{Q}_{t^{\prime}}{ }^{21,35}$ applied to an arbitrary function $\hat{F}(z)$ is

$$
\begin{aligned}
\mathcal{Q}_{t^{\prime}} \hat{F}(z)= & \hat{F}(z)-\operatorname{Tr}\left[\bar{\rho}_{t^{\prime}} \hat{F}\right]-\sum_{i}\left(\hat{A}_{i}(z)-a_{i}\left(t^{\prime}\right)\right) \\
& \times \frac{\partial}{\partial a_{i}\left(t^{\prime}\right)} \operatorname{Tr}\left[\bar{\rho}_{t^{\prime}} \hat{F}\right] .
\end{aligned}
$$

Finally, the time ordered projected propagator $G_{t^{\prime} t}$ is given by formal series

$$
\begin{aligned}
G_{t^{\prime} t} & =1+\sum_{n=1}^{\infty} \int_{t^{\prime}}^{t} d t_{1} \cdots \int_{t^{\prime}}^{t_{n-1}} d t_{n} i L \mathcal{Q}_{t_{n}} \cdots i L \mathcal{Q}_{t_{1}} \\
& =T_{-} \exp \left\{\int_{t^{\prime}}^{t} d t^{\prime \prime} i L \mathcal{Q}_{t^{\prime \prime}}\right\}
\end{aligned}
$$

Equation (13) is a closed exact equation for the time-dependent averages $a(t)$. The only assumption taken in deriving (13) is that the initial ensemble to be used in the Liouville equation is of the relevant form. That is, it is assumed that the only knowledge at the initial time is the value of the average $a(0)$ and, therefore, the least biased initial ensemble is of the relevant form (3). Therefore, the time-dependent average $a(t)$ of the relevant variables $\hat{A}(z)$ is computed with the solution of the Liouville equation $\rho_{t}(z)$ with an initial condition which is of the relevant form. The relevant ensemble is a functional of $a(t)$ through $\lambda(t)$. The kernel becomes a functional of $a(t)$ through the relevant ensemble.

Although Eq. (13) is a closed equation, it is an integrodifferential equation which is difficult to treat in general. Nevertheless, the exact transport Eq. (13) can be approximated by a memory-less equation whenever a clear separation of time scales exists between the evolution of the averages and the decay of the memory kernel. Under this assumption and the neglect of terms of order $\mathcal{O}\left(i L \hat{A}^{3}\right)$, assumed to be small due to the slowness of the relevant variables, one obtains the Markovian equation ${ }^{21}$

$$
\dot{a}_{i}(t)=v_{i}(t)+\sum_{j} D_{i j}(t) \lambda_{j}(t)
$$

where the dissipative matrix is given by the Green-Kubo formula

$$
D_{i j}(t)=\int_{0}^{\Delta t} d t^{\prime}\left\langle\mathcal{Q}_{t} i L \hat{A}_{j} \exp \left\{i L t^{\prime}\right\} \mathcal{Q}_{t} i L \hat{A}_{i}\right\rangle^{\lambda(t)}
$$

Here, $\Delta t$ is a time large compared to the decay time of the correlation integrand but short in front of the time scale of evolution of the relevant variables. The dissipative matrix depends in 
general on the relevant variables through the relevant ensemble and, as such, it is a function of time. The dissipative matrix is, to the extent that the Markov property holds, positive definite and satisfies Onsager's reciprocity. ${ }^{21}$

It is straightforward to show that the dynamic equations (19) have as a Lyapunov function the entropy (9) and, therefore, the dynamics complies with the second law of thermodynamics. Equations (19) predict the decay of any initial value of the average of the relevant variables towards its unique equilibrium values. Forced situations may be treated with the present formalism ${ }^{21}$ but we do not consider them here for simplicity.

\section{THE SYSTEM AND THE RELEVANT VARIABLES}

In this section, we will use the theory of coarse-graining as described in Sec. II for describing hydrodynamically a fluid near a solid. Consider a liquid system of $N$ monoatomic molecules described with the position and momenta of their center of mass. The molecules are allowed to move through space unrestrictedly. To avoid the issues of an infinite number of particles required in the thermodynamic limit and to make closer contact with molecular dynamics simulations, we simply assume that the system has periodic boundary conditions. Interacting with that sea of liquid molecules, there is a group of $N^{\prime}$ bonded atoms forming what we would understand at a macroscopic level as a solid object of spherical shape.

At the microscopic level, the system is described by the set of all positions $\mathbf{q}_{i}$ and momenta $\mathbf{p}_{i}=m_{i} \mathbf{v}_{i}(i=1, \ldots, N)$ of the liquid atoms plus the positions $\mathbf{q}_{i^{\prime}}$ and momenta $\mathbf{p}_{i^{\prime}}=m_{i^{\prime}} \mathbf{v}_{i^{\prime}}\left(i^{\prime}\right.$ $=1, \ldots, N^{\prime}$ ) of the atoms of the solid sphere. For compactness, we will denote the microstate in either of the following form $z$ or $q, p, q^{\prime}, p^{\prime}$. We will distinguish with a prime the labels of the atoms of the solid sphere from the unprimed labels of the liquid atoms. The microstate of the system evolves according to Hamilton's equations with a Hamiltonian given by

$$
H(z)=\sum_{i}^{N} \frac{p_{i}^{2}}{2 m_{i}}+\sum_{i^{\prime}}^{N^{\prime}} \frac{p_{i^{\prime}}^{2}}{2 m_{i^{\prime}}}+U(z),
$$

where the potential energy $U(z)$ is given by

$$
U(z)=V^{l}(q)+V^{l s}\left(q, q^{\prime}\right)+V^{s}\left(q^{\prime}\right) .
$$

We assume for simplicity a pair-wise potential energy, where $V^{l}(q)=\frac{1}{2} \sum_{i j}^{N N} \phi_{i j}^{l l}$ is the potential of interaction between liquid atoms, $V^{l s}\left(q, q^{\prime}\right)=\sum_{i i^{\prime}}^{N N^{\prime}} \phi_{i i^{\prime}}^{l s}$ is the potential of interaction between liquid atoms and solid atoms, and $V^{s}\left(q^{\prime}\right)$ $=\frac{1}{2} \sum_{i^{\prime} j^{\prime}}^{N^{\prime} N^{\prime}} \phi_{i^{\prime} j^{\prime}}^{s s}$ is the potential of interaction between the atoms of the solid object. Self interaction of the atoms is not considered, so $\phi_{i i}=0$, etc. There are no external conservative potentials acting on the system, although they can be easily introduced. We do not consider such external potentials in order to transparently discuss the issues of momentum conservation.

Note that at a microscopic level we do not have boundaries of any kind, we only have particles interacting with particles in free (periodic) space. In lab situations, typically, fluids are contained in flasks and other type of solid objects that prevent the fluid from leaking. We could model a spherical flask containing a fluid in very much the same way as we are going to treat the solid spherical particle surrounded by the (possibly infinite in extension, or periodic) fluid. A solid is regarded as a collection of bounded atoms (that is, their relative distances do not increase without bound) that are moving and vibrating. The spherical shape of the particle should be understood, of course, in a statistical sense.

\section{A. The relevant variables}

We describe the system at a coarse-grained level by selecting as relevant variables the mass and momentum density fields of the fluid and the center of mass position and momentum of the solid sphere. These are given by the following set of phase functions:

$$
\begin{array}{ll}
\hat{\rho}_{\mathbf{r}}(z)=\sum_{i}^{N} m \delta\left(\mathbf{r}-\mathbf{q}_{i}\right), & \hat{\mathbf{R}}(z)=\frac{1}{N^{\prime}} \sum_{i^{\prime}}^{N^{\prime}} \mathbf{q}_{i^{\prime}}, \\
\hat{\mathbf{g}}_{\mathbf{r}}(z)=\sum_{i}^{N} \mathbf{p}_{i} \delta\left(\mathbf{r}-\mathbf{q}_{i}\right), & \hat{\mathbf{P}}(z)=\sum_{i^{\prime}}^{N^{\prime}} \mathbf{p}_{i^{\prime}} .
\end{array}
$$

In these phase functions, the position $\mathbf{r}$ plays the role of a continuous index labeling the phase function. The position $\mathbf{r}$ may take any value in $\mathbb{R}^{3}$, or its periodic counterpart, as we do not have any restriction to the possible motion of the particles.

For the sake of simplicity, we do not include orientational degrees of freedom of the solid for the time being. Note that by selecting the center of mass variables of the solid as the only ones necessary to describe the state of the solid, we are implicitly assuming that the remaining solid degrees of freedom are much faster than the hydrodynamic fields. In particular, we assume that any elastic behaviour of the solid is so rapidly decaying towards its equilibrium state that elastic variables do not need to be included in the description. Should this assumption be violated, the resulting dynamic equations (not including these elastic variables for the solid) would probably be non-Markovian.

A word is in order about a model for the solid that is sometimes used in simulation work, where the solid is assumed to be made of "frozen" particles that act as simple generators of forces not reacting back to the presence of fluid. In this case, the solid should be modeled as a static external field acting on the fluid. The structure of the theory changes in this case as we will discuss later.

\section{B. The time derivatives of the relevant variables}

The time derivatives of the coarse variables play a fundamental role in the final structure of the dynamic equations (19). The time derivative $i L \hat{A}$ is the result of applying the Liouville operator (15) to the relevant variables. In this section, we discuss the particular form of $i L A \hat{A}$ for the case of selected CG variables (23). The action of the Liouville operator on the CG variables gives ${ }^{38}$

$$
\begin{aligned}
& i L \hat{\rho}_{\mathbf{r}}(z)=-\boldsymbol{\nabla} \cdot \hat{\mathbf{g}}_{\mathbf{r}}(z), \\
& i L \hat{\mathbf{g}}_{\mathbf{r}}(z)=-\boldsymbol{\nabla} \cdot \hat{\mathbf{K}}_{\mathbf{r}}+\hat{\mathbf{F}}_{\mathbf{r}}^{l}(z) .
\end{aligned}
$$

Here, the kinetic stress tensor is

$$
\hat{\mathbf{K}}_{\mathbf{r}}=\sum_{i}^{N} \mathbf{p}_{i} \mathbf{v}_{i} \delta\left(\mathbf{r}-\mathbf{q}_{i}\right)
$$


and the total force density $\hat{\mathbf{F}}_{\mathbf{r}}^{l}(z)$ on the liquid is defined as

$$
\hat{\mathbf{F}}_{\mathbf{r}}^{l}(z)=\sum_{i}^{N}-\frac{\partial U}{\partial \mathbf{q}_{i}} \delta\left(\mathbf{r}-\mathbf{q}_{i}\right) .
$$

We may decompose this force density into the force that the liquid exerts on the liquid plus the force that the solid exerts on the liquid, that is,

$$
\begin{aligned}
\hat{\mathbf{F}}_{\mathbf{r}}^{l}(z) & =\hat{\mathbf{F}}_{\mathbf{r}}^{l \rightarrow 1}(z)+\hat{\mathbf{F}}_{\mathbf{r}}^{s \rightarrow 1}(z), \\
\hat{\mathbf{F}}_{\mathbf{r}}^{l \rightarrow 1}(z) & =\sum_{i j}^{N N} \hat{\mathbf{F}}_{i j} \delta\left(\mathbf{r}-\mathbf{q}_{i}\right), \\
\hat{\mathbf{F}}_{\mathbf{r}}^{s \rightarrow 1}(z) & =\sum_{i j^{\prime}}^{N N^{\prime}} \hat{\mathbf{F}}_{i j^{\prime}} \delta\left(\mathbf{r}-\mathbf{q}_{i}\right),
\end{aligned}
$$

where $\hat{\mathbf{F}}_{i j^{\prime}}$ is the force that atom $j^{\prime}$ of the solid exerts on atom $i$ of the liquid. That is, $\hat{\mathbf{F}}_{\mathbf{r}}{ }^{\rightarrow}(z)$ is the microscopic force density that the liquid exerts on the liquid molecules that are around the point $\mathbf{r}$ and $\hat{\mathbf{F}}_{\mathbf{r}}^{s \rightarrow 1}(z)$ is the microscopic force density that the solid object exerts on the liquid at the point $\mathbf{r}$.

We may write the force density as

$$
\hat{\mathbf{F}}_{\mathbf{r}}^{1 \rightarrow 1}(z)=\frac{1}{2} \sum_{i j}^{N N} \hat{\mathbf{F}}_{i j}\left(\delta\left(\mathbf{r}-\mathbf{q}_{i}\right)-\delta\left(\mathbf{r}-\mathbf{q}_{j}\right)\right),
$$

where we have used that the indices are dummy. By using a standard trick, ${ }^{21,39}$ we may express the difference of the Dirac delta functions in terms of a divergence

$$
\begin{aligned}
\delta\left(\mathbf{r}-\mathbf{q}_{i}\right)-\delta\left(\mathbf{r}-\mathbf{q}_{j}\right) & =\int_{0}^{1} d \epsilon \frac{d}{d \epsilon} \delta\left(\mathbf{r}-\mathbf{q}_{j}-\epsilon \mathbf{q}_{i j}\right) \\
& =-\nabla \int_{0}^{1} d \epsilon \mathbf{q}_{i j} \delta\left(\mathbf{r}-\mathbf{q}_{j}-\epsilon \mathbf{q}_{i j}\right) .
\end{aligned}
$$

The liquid force density $\hat{\mathbf{F}}_{\mathbf{r}}^{l \rightarrow 1}(z)$ can then be expressed as the divergence of the microscopic virial stress tensor, that is,

$$
\begin{aligned}
\hat{\mathbf{F}}_{\mathbf{r}}^{l \rightarrow 1}(z) & =-\boldsymbol{\nabla} \cdot \hat{\mathbf{\Pi}}_{\mathbf{r}}(z), \\
\hat{\mathbf{\Pi}}_{\mathbf{r}}(z) & \equiv \frac{1}{2} \sum_{i j}^{N} \mathbf{q}_{i j} \hat{\mathbf{F}}_{i j} \int_{0}^{1} d \epsilon \delta\left(\mathbf{r}-\mathbf{q}_{i}+\epsilon \mathbf{q}_{i j}\right) .
\end{aligned}
$$

The time derivative of the momentum density (24) becomes

$$
i L \hat{\mathbf{g}}_{\mathbf{r}}=-\boldsymbol{\nabla} \cdot \hat{\boldsymbol{\sigma}}_{\mathbf{r}}+\mathbf{F}_{\mathbf{r}}^{\mathrm{s} \rightarrow 1},
$$

where $\hat{\sigma}_{\mathbf{r}}=\hat{\mathbf{K}}_{\mathbf{r}}+\hat{\boldsymbol{\Pi}}_{\mathbf{r}}$ is the microscopic stress tensor tensor of the fluid, that is,

$$
\begin{aligned}
\hat{\boldsymbol{\sigma}}_{\mathbf{r}}= & \sum_{i}^{N} \mathbf{p}_{i} \mathbf{v}_{i} \delta\left(\mathbf{r}-\mathbf{q}_{i}\right)+\frac{1}{2} \sum_{i j}^{N} \mathbf{q}_{i j} \hat{\mathbf{F}}_{i j} \\
& \times \int_{0}^{1} d \epsilon \delta\left(\mathbf{r}-\mathbf{q}_{i}+\epsilon \mathbf{q}_{i j}\right) .
\end{aligned}
$$

We now make the fundamental observation that the force density on the fluid (31) has two components, one which is the force done by the fluid itself and that has the form of a divergence of the stress tensor $\hat{\sigma}_{\mathbf{r}}$ and another one due to the solid $\mathbf{F}_{\mathbf{r}}^{\mathrm{s} \rightarrow 1}$ that cannot be expressed as the divergence of a stress [because the trick (29) is no longer applicable]. As we will see, because the Green-Kubo expressions for the transport coefficients involve the correlations of the fluctuations of $i L \hat{\mathbf{g}}_{\mathbf{r}}$, the decomposition (31) into stress divergence and force will lead to a very specific form of the dissipative forces on the fluid, with different space derivatives (two, one, or none) depending on the number of times the stress appears in the correlation function.

For the solid object, we have that the action of the Liouville operator gives

$$
\begin{aligned}
i L \hat{\mathbf{R}} & =\frac{\hat{\mathbf{P}}}{M}, \\
i L \hat{\mathbf{P}} & =-\int d \mathbf{r} \hat{\mathbf{F}}_{\mathbf{r}}^{s \rightarrow 1}(z) .
\end{aligned}
$$

Note that the total momentum, which is defined in terms of the coarse-grained variables as

$$
\hat{\mathbf{P}}_{T}=\int d \mathbf{r} \hat{\mathbf{g}}_{\mathbf{r}}(z)+\hat{\mathbf{P}}(z)
$$

satisfies $i L \hat{\mathbf{P}}_{T}=0$ and is, therefore, a conserved quantity of the microscopic dynamics. We have used that $\int d \mathbf{r} \boldsymbol{\nabla} \cdot \hat{\boldsymbol{\sigma}}=0$ due to Gauss theorem and the fact that at the infinite we assume there are no fluid molecules. A similar argument holds when the domain of integration is periodic.

\section{THE RELEVANT ENSEMBLE AND THE FREE ENERGY}

The relevant ensemble (3) when the coarse-grained variables are those in (23) takes the form

$$
\begin{aligned}
\bar{\rho}(z)= & \frac{1}{\Xi[\lambda]} \exp \left\{-\beta H_{N}(z)\right\} \\
& \times \exp \left\{-\beta \int d \mathbf{r}\left(\lambda_{\rho}(\mathbf{r}) \hat{\rho}_{\mathbf{r}}(z)+\lambda_{g}(\mathbf{r}) \cdot \hat{\mathbf{g}}_{\mathbf{r}}(z)\right)\right\} \\
& \times \exp \left\{-\beta \lambda_{R} \cdot \hat{\mathbf{R}}(z)-\beta \lambda_{P} \cdot \hat{\mathbf{P}}(z)\right\} .
\end{aligned}
$$

The normalization factor is the $\lambda$-dependent grand-canonical partition function defined as

$$
\begin{aligned}
\Xi[\lambda] \equiv & \sum_{N=0}^{\infty} \frac{1}{N ! h^{3 N}} \int d q d p d q^{\prime} d p^{\prime} \\
& \times \exp \left\{-\beta H_{N}-\beta \sum_{i=1}^{N} m \lambda_{\rho}\left(\mathbf{r}_{i}\right)-\beta \sum_{i=1}^{N} \mathbf{p}_{i} \cdot \lambda_{g}\left(\mathbf{q}_{i}\right)\right\} \\
& \times \exp \left\{-\beta \lambda_{R} \cdot \hat{\mathbf{R}}(z)-\beta \lambda_{P} \cdot \hat{\mathbf{P}}(z)\right\} .
\end{aligned}
$$

Note that for the trace in phase space we are using a macrocanonical trace concerning the fluid degrees of freedom and a canonical trace for the solid degrees of freedom. The use of the macrocanonical ensemble for the liquid is standard in DFT. In the present work, the canonical ensemble for the fluid would lead to a matrix of second derivatives (8) that, due to number of particles conservation, has a zero eigenvalue leading to an ill-defined inverse, and the argument after Eq. (8) would not strictly apply. Although this may be addressed by restricting the functional space of the density fields, the problem is happily absent in the macrocanonical ensemble. The conjugate fields $\lambda$ of the relevant variables (23) are fixed by the condition that the averages of the relevant variables (23) with the 
relevant ensemble coincide with the averages $\rho(\mathbf{r}), \mathbf{g}(\mathbf{r}), \mathbf{R}$, and $\mathbf{P}$ computed with the solution of the Liouville equation (we omit the time dependence for simplicity). This condition can be expressed as in Eqs. (6) as

$$
\begin{array}{ll}
\rho(\mathbf{r})=\frac{\delta \Phi[\lambda]}{\delta \lambda_{\rho}(\mathbf{r})}, & \mathbf{R}=\frac{\partial \Phi[\lambda]}{\partial \lambda_{R}}, \\
\mathbf{g}(\mathbf{r})=\frac{\delta \Phi[\lambda]}{\delta \lambda_{g}(\mathbf{r})}, & \mathbf{P}=\frac{\partial \Phi[\lambda]}{\partial \lambda_{P}},
\end{array}
$$

where the $\lambda$-dependent grand-canonical potential is given by

$$
\Phi[\lambda] \equiv-k_{B} T \ln \Xi[\lambda] .
$$

Because the functional $\Phi[\lambda]$ is convex, the conjugate variables $\lambda=\left\{\lambda_{\rho}, \lambda_{g}, \lambda_{R}, \lambda_{P}\right\}$ are in one to one connection with $a=\{\rho$, $\mathbf{g}, \mathbf{R}, \mathbf{P}\}$. Therefore, the functionals $\lambda_{\rho}[\rho, \mathbf{g}, \mathbf{R}, \mathbf{P}], \lambda_{g}[\rho, \mathbf{g}$, $\mathbf{R}, \mathbf{P}], \lambda_{R}[\rho, \mathbf{g}, \mathbf{R}, \mathbf{P}]$, and $\lambda_{P}[\rho, \mathbf{g}, \mathbf{R}, \mathbf{P}]$ exist and are unique. We can therefore switch from the conjugate variables $\lambda$ to the relevant variables $a$ and construct the corresponding hydrodynamic functional. The hydrodynamic functional is given by the Legendre transform of the $\lambda$-dependent grand canonical potential, that is,

$$
\begin{aligned}
\mathcal{H}[\rho, \mathbf{g}, \mathbf{R}, \mathbf{P}]= & \Phi\left[\lambda_{\rho}, \lambda_{g}, \lambda_{R}, \lambda_{P}\right]-\int d \mathbf{r} \rho(\mathbf{r}) \lambda_{\rho}(\mathbf{r}) \\
& -\int d \mathbf{r g}(\mathbf{r}) \cdot \lambda_{g}(\mathbf{r})-\lambda_{R} \cdot \mathbf{R}-\lambda_{P} \cdot \mathbf{P},
\end{aligned}
$$

where here the conjugate fields $\lambda$ are to be understood as functionals of the average fields $a$. The hydrodynamic functional is the negative of the corresponding entropy (9) for the present level of description. Being a Legendre transform, the hydrodynamic functional satisfies the following relationships [see Eq. (11)], conjugate of those in Eq. (23):

$$
\begin{array}{ll}
\lambda_{\rho}(\mathbf{r})=-\frac{\delta \mathcal{H}}{\delta \rho(\mathbf{r})}, & \lambda_{R}=-\frac{\partial \mathcal{H}}{\partial \mathbf{R}}, \\
\lambda_{g}(\mathbf{r})=-\frac{\delta \mathcal{H}}{\delta \mathbf{g}(\mathbf{r})}, & \lambda_{P}=-\frac{\partial \mathcal{H}}{\partial \mathbf{P}} .
\end{array}
$$

In fact, it is possible to find the explicit expression of $\lambda_{g}$ and $\lambda_{P}$ by performing the momentum integrals in Eq. (36). One obtains

$$
\begin{aligned}
\Xi[\lambda] \equiv & \sum_{N=0}^{\infty} \frac{1}{N !} \int \frac{d q}{\Lambda^{3 N}} \frac{d q^{\prime}}{\Lambda^{3 N^{\prime}}} e^{-\beta U} \\
& \times \exp \left\{-\beta \sum_{i=1}^{N}\left(m \lambda_{\rho}\left(\mathbf{r}_{i}\right)-\frac{m}{2} \lambda_{g}^{2}\left(\mathbf{q}_{i}\right)\right)\right\} \\
& \times \exp \left\{-\beta\left(\lambda_{R} \cdot \hat{\mathbf{R}}-\frac{M^{\prime}}{2} \lambda_{P}^{2}\right)\right\}
\end{aligned}
$$

where $\Lambda$ is the thermal wavelength. Then take the functional derivative of Eq. (41) with respect to the conjugate field $\lambda_{g}(\mathbf{r})$, and the derivative with respect to $\lambda_{P}$. Together with Eqs. (37) and (38), this leads directly to the explicit form of the following conjugate variables:

$$
\begin{aligned}
\lambda_{g}(\mathbf{r}) & =-\frac{\mathbf{g}(\mathbf{r})}{\rho(\mathbf{r})}=-\mathbf{v}(\mathbf{r}), \\
\lambda_{P} & =-\frac{\mathbf{P}}{M^{\prime}},
\end{aligned}
$$

and allows one to interpret these conjugate variables as (negative) velocities. The grand potential (38) takes now the form

$$
\Phi[\lambda]=\Phi^{\mathrm{pos}}\left[\mu, \lambda_{R}\right]-\frac{M^{\prime}}{2} \lambda_{P}^{2},
$$

where we have defined the following grand potential:

$$
\begin{aligned}
\Phi^{\mathrm{pos}}\left[\mu, \lambda_{R}\right] \equiv & -k_{B} T \ln \sum_{N=0}^{\infty} \frac{1}{N !} \int \frac{d q}{\Lambda^{3 N}} \frac{d q^{\prime}}{\Lambda^{3 N^{\prime}}} \\
& \times \exp \left\{-\beta\left(U-\sum_{i=1}^{N} m \mu\left(\mathbf{r}_{i}\right)+\lambda_{R} \cdot \hat{\mathbf{R}}\right)\right\},
\end{aligned}
$$

and the chemical potential per unit mass has been introduced as

$$
\mu(\mathbf{r}) \equiv \frac{1}{2} \lambda_{g}^{2}(\mathbf{r})-\lambda_{\rho}(\mathbf{r})
$$

The grand potential $\Phi^{\mathrm{pos}}\left[\mu, \lambda_{R}\right]$ is similar to the macrocanonical grand potential of a fluid, except for the presence of the solid degrees of freedom and the corresponding conjugate variable $\lambda_{R}$. The Legendre transform of the grand potential for a simple liquid gives the classic (free energy) density functional and we may pursue now the same route in order to define the free energy density functional for a fluid in the presence of a solid sphere. We need first to compute the derivatives of the functional (44) that give

$$
\begin{aligned}
-\frac{\delta \Phi^{\mathrm{pos}}}{\delta \mu(\mathbf{r})}\left[\mu, \lambda_{R}\right] & =\left\langle\hat{\rho}_{\mathbf{r}}\right\rangle^{\mu, \lambda_{R},}, \\
\frac{\partial \Phi^{\mathrm{pos}}}{\partial \lambda_{R}}\left[\mu, \lambda_{R}\right] & =\langle\hat{\mathbf{R}}\rangle^{\mu, \lambda_{R}},
\end{aligned}
$$

where the averages $\langle\cdots\rangle^{\mu, \lambda_{R}}$ are defined in these equations. The second derivatives of $\Phi^{\text {pos }}$ are given by covariances [see Eqs. (6)-(8)] and this implies that $\Phi^{\mathrm{pos}}\left[\mu, \lambda_{R}\right]$ is a convex function. Therefore, the connection between $\left\langle\hat{\rho}_{\mathbf{r}}\right\rangle^{\mu, \lambda_{R}},\langle\hat{\mathbf{R}}\rangle^{\mu, \lambda_{R}}$ and $\mu(\mathbf{r}), \lambda_{R}$ is one to one. Note also that because the phase functions $\hat{\rho}_{\mathbf{r}}, \hat{\mathbf{R}}$ do not depend on particle's momenta, we have that the averages are given by

$$
\begin{aligned}
\left\langle\hat{\rho}_{\mathbf{r}}\right)^{\mu, \lambda_{R}} & =\operatorname{Tr}\left[\bar{\rho} \hat{\rho}_{\mathbf{r}}\right]=\rho_{\mathbf{r}}, \\
\langle\hat{\mathbf{R}}\rangle^{\mu, \lambda_{R}} & =\operatorname{Tr}[\bar{\rho} \hat{\mathbf{R}}]=\mathbf{R} .
\end{aligned}
$$

Therefore, we have a one to one connection between the conjugate variables $\mu(\mathbf{r}), \lambda_{R}$ and the averages $\rho(\mathbf{r}), \mathbf{R}$ of the $\mathrm{CG}$ variables. The free energy functional $\mathcal{F}[\rho, \mathbf{R}]$ of a structured fluid in the presence of a solid sphere is obtained as the following Legendre transform:

$$
\mathcal{F}[\rho, \mathbf{R}] \equiv \Phi^{\mathrm{pos}}\left[\mu, \lambda_{R}\right]+\int d \mathbf{r} \rho(\mathbf{r}) \mu(\mathbf{r})-\lambda_{R} \cdot \mathbf{R},
$$

whose derivatives are given by

$$
\begin{aligned}
\frac{\delta \mathcal{F}}{\delta \rho(\mathbf{r})}[\rho, \mathbf{R}] & =\mu(\mathbf{r}, \mathbf{R}), \\
\frac{\partial \mathcal{F}}{\partial \mathbf{R}}[\rho, \mathbf{R}] & =-\lambda_{R} .
\end{aligned}
$$

We may now express the hydrodynamic functional (39) as

$$
\mathcal{H}[\rho, \mathbf{g}, \mathbf{R}, \mathbf{P}]=\int d \mathbf{r} \frac{\mathbf{g}^{2}(\mathbf{r})}{2 \rho(\mathbf{r})}+\frac{\mathbf{P}^{2}}{2 M^{\prime}}+\mathcal{F}[\rho, \mathbf{R}] .
$$


The expression (50) of the hydrodynamic functional $\mathcal{H}$ as the sum of a kinetic part and a "potential" part that depends on both the density and the position of the solid is a non-trivial exact result.

We may compute the functional derivative of the hydrodynamic functional (50) with respect to the position $\mathbf{R}$ and we obtain

$$
\frac{\partial \mathcal{H}}{\partial \mathbf{R}}[\rho, \mathbf{g}, \mathbf{R}, \mathbf{P}]=\frac{\partial \mathcal{F}}{\partial \mathbf{R}}[\rho, \mathbf{R}]=-\lambda_{R} .
$$

As we will see in Appendix A, $\lambda_{R}$ is just the average force on the solid due to the fluid. Therefore, the "potential" part $\mathcal{F}[\rho, \mathbf{R}]$ of the hydrodynamic functional (39) really acts as a potential energy whose negative gradient gives the actual force on the sphere. For future reference, we may also compute the functional derivative of the hydrodynamic functional $\mathcal{H}$ with respect to the density field with the result

$$
\frac{\delta \mathcal{H}}{\delta \rho(\mathbf{r})}[\rho, \mathbf{g}, \mathbf{R}, \mathbf{P}]=-\frac{\mathbf{v}^{2}(\mathbf{r})}{2}+\frac{\delta \mathcal{F}}{\delta \rho(\mathbf{r})}[\rho, \mathbf{R}] .
$$

The free energy functional is translationally invariant, that is,

$$
\mathcal{F}\left[T_{\mathbf{u}} \rho, T_{\mathbf{u}} \mathbf{R}\right]=\mathcal{F}[\rho, \mathbf{R}],
$$

where the translation operator applied to any function is defined as

$$
T_{\mathbf{u}} f(\mathbf{r})=f(\mathbf{r}+\mathbf{u}) .
$$

The invariance can be shown by performing a suitable change of variables inside the phase space integrals defining the partition function. By taking the derivative with respect to $\mathbf{u}$ in both sides of Eq. (53) and setting afterwards $\mathbf{u}=0$, we obtain the identity

$$
\int d \mathbf{r} \frac{\delta \mathcal{F}}{\delta \rho_{\mathbf{r}}} \nabla \rho_{\mathbf{r}}+\frac{\partial \mathcal{F}}{\partial \mathbf{R}}=0 .
$$

This identity will be crucial in order to show that total momentum is conserved by the reversible part of the dynamics.

The calculation of the thermodynamic potential (44) needed for the evaluation of the free energy functional $\mathcal{F}[\rho, \mathbf{R}]$ is very difficult to perform in general and, therefore, one needs to model the free energy functional based on intuition and previous experience. A particularly simple model that takes into account the effect of the fluid-solid interaction is given by

$$
\mathcal{F}[\rho, \mathbf{R}]=\mathcal{F}_{0}[\rho]+\int d \mathbf{r} \frac{1}{m} \rho(\mathbf{r}) V(\mathbf{r}, \mathbf{R}),
$$

where $\mathcal{F}_{0}[\rho]$ is the free energy density functional of the fluid in the absence of the solid, and all the fluid solid interaction is taken into account through the second term that involves a coarse-grained potential $V(\mathbf{r}, \mathbf{R})$. This potential captures the effective (reversible) interaction between solid and fluid atoms. Note that even for the case of an ideal gas interacting with a solid sphere, the model (56) does not follow straightforwardly from the exact free energy (48) with (44) due to the integrals over the solid degrees of freedom of the interaction potential between ideal gas particles and solid particles. Eq. (56) is just a natural candidate to model the free energy $F[\rho, \mathbf{R}]$. The potential energy $V(\mathbf{r}, \mathbf{R})$ becomes infinite (or extremely large) for the points $\mathbf{r}$ inside the solid sphere. Therefore, the last term in (56) makes impossible the realization of density fields with non-zero value inside the solid sphere (i.e., leads to impenetrability of the solid). The derivatives of the free energy model (56) are

$$
\begin{aligned}
\frac{\delta \mathcal{F}}{\delta \rho(\mathbf{r})} & =\mu_{0}(\mathbf{r})+V(\mathbf{r}, \mathbf{R}), \\
\frac{\partial \mathcal{F}}{\partial \mathbf{R}} & =-\int d \mathbf{r} \rho(\mathbf{r}) \mathbf{F}(\mathbf{r}, \mathbf{R}),
\end{aligned}
$$

where $\mu_{0}(\mathbf{r})$ is the usual chemical potential of the solvent in the absence of any solid, and $\mathbf{F}(\mathbf{r}, \mathbf{R})$ is the effective force, deriving from the potential $V(\mathbf{r}, \mathbf{R})$ that the solid sphere with center of mass at $\mathbf{R}$ exerts on a fluid atom at $\mathbf{r}$.

\section{TOWARDS THE TRANSPORT EQUATIONS}

\section{A. Exact reversible dynamics}

We consider in this subsection the reversible part $v_{i}(t)$ in Eq. (14) for the case that the CG variables are those in Eqs. (23). For the mass density, we have

$$
\left.\partial_{t} \rho(\mathbf{r}, t)\right|_{\mathrm{rev}}=\operatorname{Tr}\left[\bar{\rho}_{t} i L \hat{\rho}_{\mathbf{r}}\right]=-\boldsymbol{\nabla} \cdot \mathbf{g}(\mathbf{r}, t),
$$

where we have used Eq. (24) and the fact that the relevant ensemble average of $\hat{\mathbf{g}}_{\mathbf{r}}$ is precisely the momentum density field $\mathbf{g}(\mathbf{r}, t)$. On the other hand, the reversible part of the momentum density evolution equation is

$$
\begin{aligned}
\left.\partial_{t} \mathbf{g}(\mathbf{r}, t)\right|_{\text {rev }} & =\operatorname{Tr}\left[\bar{\rho}_{t} i L \hat{\mathbf{g}}_{\mathbf{r}}\right] \\
& =-\nabla \cdot \operatorname{Tr}\left[\bar{\rho}_{t} \hat{\mathbf{K}}_{\mathbf{r}}\right]+\operatorname{Tr}\left[\bar{\rho}_{t} \hat{\mathbf{F}}_{\mathbf{r}}^{\mathrm{l}}\right] .
\end{aligned}
$$

We introduce the Galilean operator $\mathcal{G}$ that when applied to any phase function changes its velocity arguments from $\mathbf{v}_{i} \rightarrow \mathbf{v}_{i}$ $+\mathbf{v}\left(\mathbf{q}_{i}\right)$ for $i$ a fluid particle, that is,

$$
\begin{aligned}
\mathcal{G} \hat{F} & \left(\mathbf{q}_{1}, \ldots, \mathbf{q}_{N}, \mathbf{p}_{1}, \ldots, \mathbf{p}_{N}\right) \\
\quad & =\hat{F}\left(\mathbf{q}_{1}, \ldots, \mathbf{q}_{N}, \mathbf{p}_{1}+m_{1} \mathbf{v}\left(\mathbf{q}_{1}\right), \ldots, \mathbf{p}_{N}+m_{N} \mathbf{v}\left(\mathbf{q}_{N}\right)\right) .
\end{aligned}
$$

Within any trace, this is just a change of variables. Therefore, we have the property

$$
\operatorname{Tr}\left[\bar{\rho}_{t} \hat{F}\right]=\operatorname{Tr}\left[\left(\mathcal{G} \bar{\rho}_{t}\right)(\mathcal{G} \hat{F})\right] .
$$

The action of the Galilean operator on the relevant ensemble is

$$
\begin{aligned}
\mathcal{G} \bar{\rho}_{t}= & \frac{1}{\Xi[\lambda(t)]} \rho^{\mathrm{eq}}(z) \exp \left\{\beta \int d \mathbf{r} \mu(\mathbf{r}) \hat{\rho}_{\mathbf{r}}(z)\right\} \\
& \times \exp \left\{-\beta \lambda_{R}(t) \cdot \hat{\mathbf{R}}-\beta \boldsymbol{\lambda}_{P}(t) \cdot \hat{\mathbf{P}}\right\},
\end{aligned}
$$

where the chemical potential per unit mass has been introduced in Eq. (45). The action of the Galilean operator on the microscopic kinetic stress tensor is

$$
\mathcal{G} \hat{\mathbf{K}}_{\mathbf{r}}=\hat{\mathbf{K}}_{\mathbf{r}}+\mathbf{v}(\mathbf{r}) \hat{\mathbf{g}}_{\mathbf{r}}+\hat{\mathbf{g}}_{\mathbf{r}} \mathbf{v}(\mathbf{r})+\mathbf{v}(\mathbf{r}) \mathbf{v}(\mathbf{r}) \hat{\rho}_{\mathbf{r}}
$$

By noting that the ensemble (62) is Gaussian in momenta, we have finally

$$
\operatorname{Tr}\left[\bar{\rho}_{t} \hat{\mathbf{K}}_{\mathbf{r}}\right]=\mathbf{v}(\mathbf{r}) \mathbf{v}(\mathbf{r}) \rho(\mathbf{r})+\frac{k_{B} T}{m} \rho(\mathbf{r}) \boldsymbol{\delta},
$$

where $\boldsymbol{\delta}$ is the unit tensor. The last term $\operatorname{Tr}\left[\bar{\rho}_{t} \hat{\mathbf{F}}_{\mathbf{r}}\right]$ in Eq. (59) is computed in Eq. (A8) of Appendix A. By collecting (64) and (A8) into the momentum density equation (59), we obtain

$$
\begin{aligned}
\left.\partial_{t} \mathbf{g}(\mathbf{r}, t)\right|_{\mathrm{rev}}= & -\boldsymbol{\nabla} \cdot(\mathbf{v}(\mathbf{r}, t) \mathbf{g}(\mathbf{r}, t)) \\
& -\rho(\mathbf{r}) \boldsymbol{\nabla} \frac{\delta \mathcal{F}}{\delta \rho(\mathbf{r})}[\rho, \mathbf{R}] .
\end{aligned}
$$


Finally, the averages with the relevant ensemble of the solid object variables are

$$
\begin{aligned}
\operatorname{Tr}\left[\bar{\rho}_{t} i L \hat{\mathbf{R}}\right] & =\frac{\mathbf{P}}{M}, \\
\operatorname{Tr}\left[\bar{\rho}_{t} i L \hat{\mathbf{P}}\right] & =-\frac{\partial \mathcal{F}}{\partial \mathbf{R}}[\rho, \mathbf{R}],
\end{aligned}
$$

where we have used Eq. (A10) in Appendix A.

By collecting the above results, the reversible part of the dynamics has the form

$$
\begin{aligned}
\left.\partial_{t} \rho(\mathbf{r})\right|_{\text {rev }} & =-\boldsymbol{\nabla} \cdot \mathbf{g}(\mathbf{r}), \\
\left.\partial_{t} \mathbf{g}(\mathbf{r})\right|_{\mathrm{rev}} & =-\boldsymbol{\nabla} \cdot(\mathbf{g}(\mathbf{r}) \mathbf{v}(\mathbf{r}))-\rho(\mathbf{r}) \boldsymbol{\nabla} \frac{\delta \mathcal{F}}{\delta \rho(\mathbf{r})}[\rho, \mathbf{R}], \\
\left.\partial_{t} \mathbf{R}\right|_{\text {rev }} & =\frac{\mathbf{P}}{M}, \\
\left.\partial_{t} \mathbf{P}\right|_{\text {rev }} & =-\frac{\partial \mathcal{F}}{\partial \mathbf{R}}[\rho, \mathbf{R}] .
\end{aligned}
$$

These reversible equations are exact as no approximations have been made so far. We qualify these equations as reversible because, as can be explicitly shown, they conserve the hydrodynamic functional (39), which is minus the entropy corresponding to this level of description.

\section{B. Markovian irreversible dynamics}

While the reversible part of the dynamics (67) is exact, the irreversible part that we consider in this paper is approximate because we will assume a Markovian dynamics. Under the Markovian approximation in which the memory kernel is assumed to decay in a time scale short as compared to the time scales of the hydrodynamic variables, the irreversible dynamics is given by the term $\sum_{j} D_{i j} \lambda_{j}$ in Eq. (19). Because the time derivatives of $\rho(\mathbf{r})$ and $\mathbf{R}$ are given in terms of momenta, which are relevant variables themselves, the effect of the projection operator in (17) is simply $\mathcal{Q} i L \rho_{\mathbf{r}}=0$ and $\mathcal{Q} i L \mathbf{r}_{\mu}=0$ resulting in a large simplification of the friction matrix. The irreversible part of the dynamics $\sum_{j} D_{i j} \lambda_{j}$ in Eq. (19) now takes the form

$$
\partial_{t}\left(\begin{array}{c}
\rho(\mathbf{r}) \\
\mathbf{g}(\mathbf{r}) \\
\mathbf{R} \\
\mathbf{p}
\end{array}\right)_{\mathrm{irr}}=-\left(\begin{array}{cccc}
0 & 0 & 0 & 0 \\
0 & \int d \mathbf{r}^{\prime} M_{\mathbf{r r}^{\prime}}^{g g} & 0 & M_{\mathbf{r}}^{g P} \\
0 & 0 & 0 & 0 \\
0 & \int d \mathbf{r}^{\prime} M_{\mathbf{r}^{\prime}}^{P g} & 0 & M^{P P}
\end{array}\right)\left(\begin{array}{c}
\frac{\delta \mathcal{H}}{\delta \rho_{\mathbf{r}^{\prime}}} \\
\frac{\delta \mathcal{H}}{\delta \mathbf{g}_{\mathbf{r}^{\prime}}} \\
\frac{\partial \mathcal{H}}{\delta \mathbf{R}} \\
\frac{\partial \mathcal{H}}{\delta \mathbf{p}}
\end{array}\right),
$$

where we have used (40). The sum over the continuum "index" $\mathbf{r}$ becomes an integral. The domain of integration of this integral is $\mathbb{R}^{3}$, including the interior of the solid sphere. By using the results (40) and (42) that link the functional derivatives of the CG Hamiltonian with respect to momenta to the velocities, we obtain the following irreversible dynamics:

$$
\begin{aligned}
\partial_{t} \mathbf{g}(\mathbf{r}) & =-\int d \mathbf{r}^{\prime} M_{\mathbf{r} \mathbf{r}^{\prime}}^{g g} \mathbf{v}\left(\mathbf{r}^{\prime}\right)-M_{\mathbf{r}}^{g P} \mathbf{V}, \\
\frac{d}{d t} \mathbf{p}(t) & =-\int d \mathbf{r}^{\prime} M_{\mathbf{r}^{\prime}}^{P g} \mathbf{v}\left(\mathbf{r}^{\prime}\right)-M^{P P} \mathbf{V},
\end{aligned}
$$

where the matrix elements are defined in terms of Green-Kubo formulae as

$$
\begin{aligned}
M_{\mathbf{r} \mathbf{r}^{\prime}}^{g g} & =\frac{1}{k_{B} T} \int_{0}^{\Delta t} d t\left\langle\mathcal{Q} i L \hat{\mathbf{g}}_{\mathbf{r}}(t) \mathcal{Q} i L \hat{\mathbf{g}}_{\mathbf{r}^{\prime}}\right\rangle^{\lambda}, \\
M_{\mathbf{r}}^{g P} & =\frac{1}{k_{B} T} \int_{0}^{\Delta t} d t\left\langle\mathcal{Q} i L \hat{\mathbf{g}}_{\mathbf{r}}(t) \mathcal{Q} i L \hat{\mathbf{P}}\right\rangle^{\lambda}, \\
M_{\mathbf{r}^{\prime}}^{P g} & =\frac{1}{k_{B} T} \int_{0}^{\Delta t} d t\left\langle\mathcal{Q} i L \hat{\mathbf{P}}(t) \mathcal{Q} i L \hat{\mathbf{g}}_{\mathbf{r}^{\prime}}\right\rangle^{\lambda}, \\
M^{P P} & =\frac{1}{k_{B} T} \int_{0}^{\Delta t} d t\langle\mathcal{Q} i L \hat{\mathbf{P}}(t) \mathcal{Q} i L \hat{\mathbf{P}}\rangle^{\lambda}
\end{aligned}
$$

Note that momentum conservation implies that the gain rate of the total momentum of the fluid is equal to the loss rate of momentum of the particle

$$
\int d \mathbf{r} i L \hat{\mathbf{g}}_{\mathbf{r}}=-i L \hat{\mathbf{P}} .
$$

The conservation of momenta then implies the following relationships between elements of the dissipative matrix:

$$
\begin{aligned}
& M_{\mathbf{r}}^{g P}=-\int d \mathbf{r}^{\prime} M_{\mathbf{r r}}^{g g}, \\
& M^{P P}=-\int d \mathbf{r} M_{\mathbf{r}}^{P g},
\end{aligned}
$$

and this leads in Eq. (69) to

$$
\begin{aligned}
& \partial_{t} \mathbf{g}(\mathbf{r})=-\int d \mathbf{r}^{\prime} M_{\mathbf{r \mathbf { r } ^ { \prime }}}^{g g}\left(\mathbf{v}\left(\mathbf{r}^{\prime}\right)-\mathbf{V}\right), \\
& \frac{d}{d t} \mathbf{p}(t)=\int d \mathbf{r} \int d \mathbf{r}^{\prime} M_{\mathbf{r \mathbf { r } ^ { \prime }}}^{g g}\left(\mathbf{v}\left(\mathbf{r}^{\prime}\right)-\mathbf{V}\right),
\end{aligned}
$$

which contain only the matrix element $M_{\mathbf{r r}^{\prime}}^{g g}$ and are manifestly Galilean invariant. By using Eqs. (31) and (33) in the definition of $M_{\mathbf{r r}^{\prime}}^{g g}$ in Eq. (70) and inserting the result in the equation of motion (69), one obtains

$$
\begin{aligned}
\left.\partial_{t} \mathbf{g}^{\alpha}(\mathbf{r})\right|_{\mathrm{irr}} & =\nabla_{\mathbf{r}}^{\beta} \boldsymbol{\Sigma}^{\alpha \beta}(\mathbf{r})+\mathcal{S}^{\alpha}(\mathbf{r}), \\
\left.\frac{d}{d t} \mathbf{P}^{\alpha}(t)\right|_{\mathrm{irr}} & =-\int d \mathbf{r}^{\prime} \mathcal{S}^{\alpha}\left(\mathbf{r}^{\prime}\right),
\end{aligned}
$$

where the fluid stress tensor is

$$
\Sigma^{\alpha \beta}(\mathbf{r})=\int d \mathbf{r}^{\prime} \eta_{\mathbf{r r}^{\prime}}^{\alpha \beta \alpha^{\prime} \beta^{\prime}} \nabla_{\mathbf{r}^{\prime}}^{\beta^{\prime}} \mathbf{v}^{\alpha^{\prime}}\left(\mathbf{r}^{\prime}\right),
$$

and the irreversible surface force density on the fluid is defined as

$$
\begin{aligned}
\mathcal{S}^{\alpha}(\mathbf{r})= & -\int d \mathbf{r}^{\prime} \mathbf{G}_{\mathbf{r} \mathbf{r}^{\prime}}^{\alpha \beta^{\prime}} \nabla_{\mathbf{r}^{\prime}}^{\beta^{\prime} \mathbf{v}^{\alpha^{\prime}}\left(\mathbf{r}^{\prime}\right)} \\
& +\nabla_{\mathbf{r}}^{\beta} \int d \mathbf{r}^{\prime} \mathbf{H}_{\mathbf{r r}^{\prime}}^{\alpha \beta \alpha^{\prime}}\left(\mathbf{v}^{\alpha^{\prime}}\left(\mathbf{r}^{\prime}\right)-\mathbf{V}^{\alpha^{\prime}}\right) \\
& -\int d \mathbf{r}^{\prime} \gamma_{\mathbf{r r}^{\prime}}^{\alpha \alpha^{\prime}}\left(\mathbf{v}^{\alpha^{\prime}}\left(\mathbf{r}^{\prime}\right)-\mathbf{V}^{\alpha^{\prime}}\right)
\end{aligned}
$$

We use Einstein's sum over repeated indices convention. In these expressions, we have introduced the following non-local transport coefficients: the viscosity kernel $\boldsymbol{\eta}_{\mathbf{r r}^{\prime}}$ (fourth order tensor), the slip kernels $\mathbf{H}_{\mathbf{r r}}, \mathbf{G}_{\mathbf{r r}^{\prime}}$ (third order tensors), and the friction kernel $\gamma_{\mathbf{r r}^{\prime}}$ (second order tensor). They are defined by the Green-Kubo formulae 


$$
\begin{aligned}
\eta_{\mathbf{r r}} & \equiv \frac{1}{k_{B} T} \int_{0}^{\Delta t} d t^{\prime}\left\langle\mathcal{Q}_{t} \hat{\boldsymbol{\sigma}}_{\mathbf{r}}\left(t^{\prime}\right) \mathcal{Q}_{t} \hat{\boldsymbol{\sigma}}_{\mathbf{r}^{\prime}}\right\rangle^{\lambda(t)}, \\
\mathbf{H}_{\mathbf{r} \mathbf{r}^{\prime}} & \equiv \frac{1}{k_{B} T} \int_{0}^{\Delta t} d t^{\prime}\left\langle\mathcal{Q}_{t} \hat{\boldsymbol{\sigma}}_{\mathbf{r}}\left(t^{\prime}\right) \mathcal{Q}_{t} \hat{\mathbf{F}}_{\mathbf{r}^{\prime}}^{\mathrm{s} \rightarrow 1}\right\rangle^{\lambda(t)}, \\
\mathbf{G}_{\mathbf{r r}^{\prime}} & \equiv \frac{1}{k_{B} T} \int_{0}^{\Delta t} d t^{\prime}\left\langle\mathcal{Q}_{t} \hat{\mathbf{F}}_{\mathbf{r}}^{s \rightarrow 1}\left(t^{\prime}\right) \mathcal{Q}_{t} \hat{\boldsymbol{\sigma}}_{\mathbf{r}^{\prime}}\right\rangle^{\lambda(t)}, \\
\gamma_{\mathbf{r} \mathbf{r}^{\prime}} & \equiv \frac{1}{k_{B} T} \int_{0}^{\Delta t} d t^{\prime}\left\langle\mathcal{Q}_{t} \hat{\mathbf{F}}_{\mathbf{r}}^{\mathrm{s} \rightarrow 1}\left(t^{\prime}\right) \mathcal{Q}_{t} \hat{\mathbf{F}}_{\mathbf{r}^{\prime}}^{\mathrm{s} \rightarrow 1}\right\rangle^{\lambda(t)} .
\end{aligned}
$$

These transport kernels are state dependent, i.e., functions of the time-dependent averages of the relevant variables, through the dependence of the relevant ensemble on these averages. The explicit form of the projected currents appearing in these expressions is given in Appendix B.

\section{THE FINAL EQUATIONS OF NANO-HYDRODYNAMICS}

By collecting the reversible part of the dynamics (67) and the irreversible part (74), we obtain the final dynamic equations for the relevant variables

$$
\begin{aligned}
\partial_{t} \rho(\mathbf{r})= & -\boldsymbol{\nabla} \cdot \mathbf{g}(\mathbf{r}), \\
\partial_{t} \mathbf{g}(\mathbf{r})= & -\boldsymbol{\nabla} \cdot(\mathbf{g}(\mathbf{r}) \mathbf{v}(\mathbf{r}))-\rho(\mathbf{r}) \boldsymbol{\nabla} \frac{\delta \mathcal{F}}{\delta \rho(\mathbf{r})}[\rho, \mathbf{R}] \\
& +\boldsymbol{\nabla} \cdot \boldsymbol{\Sigma}(\mathbf{r})+\mathcal{S}(\mathbf{r}), \\
\dot{\mathbf{R}}= & \frac{\mathbf{P}}{M}, \\
\dot{\mathbf{P}}= & -\frac{\partial \mathcal{F}}{\partial \mathbf{R}}-\int d \mathbf{r} \mathcal{S}(\mathbf{r}),
\end{aligned}
$$

where the free energy functional $\mathcal{F}[\rho, \mathbf{R}]$ is introduced in Eq. (48), the velocity field is $\mathbf{v}(\mathbf{r})=\mathbf{g}(\mathbf{r}) / \rho(\mathbf{r})$, the fluid stress tensor $\boldsymbol{\Sigma}(\mathbf{r})$ is given in (75), and the irreversible force $\mathcal{S}(\mathbf{r})$ is given in Eq. (76).

Eqs. (78), (77), and (76) are the main results of the present paper. They describe the isothermal non-local hydrodynamics of a simple fluid coupled with the motion of an immersed structureless solid sphere. These equations generalize equilibrium density functional theory for a simple fluid to non-equilibrium situations in which the fluid may be moving around a solid sphere. The only approximation that has been taken in the derivation is the Markovian approximation that neglects memory effects in the dissipative part of the dynamics.

Let us discuss the physical meaning of the different terms in Eqs. (78). The equation for the evolution of the mass density field is just the continuity equation. The equation for the momentum density field involves the usual convective term plus a term involving the gradient of the functional derivative of the free energy. This term describes both the "pressure gradient" term and the reversible coupling between the fluid and solid sphere. The usual pressure gradient term of the NavierStokes equations is obtained when one uses a local free energy functional as will be shown elsewhere. The viscous term $\nabla \cdot \boldsymbol{\Sigma}$ in Eqs. (78) describes the internal friction of the fluid due to its self-interaction. This viscous term involving second derivatives is the usual viscosity term of the Navier-Stokes equations, which is here expressed in a non-local form. The use of nonlocal viscosities has been advocated recently in the field of nano-hydrodynamics. ${ }^{23-26}$ A phenomenological alternative to address flow problems where density is inhomogeneous is to keep using local hydrodynamics with the viscosity of the bulk but evaluated at the local values of the density. ${ }^{40}$ While this may lead to reasonable results (but not always ${ }^{41}$ ), the present microscopically derived model seems to have a firmer basis. The fourth-order viscosity tensor is given in terms of the correlation of the fluctuations of the fluid stress tensor. Away from the walls, it is expected that the viscosity tensor becomes fully isotropic and dependent only on the distance between the points $\mathbf{r}$ and $\mathbf{r}^{\prime}$.

The force density $\mathcal{S}(\mathbf{r})$ involving the non-local transport kernels $\mathbf{H}_{\mathbf{r r}^{\prime}}, \mathbf{G}_{\mathbf{r r}^{\prime}}, \boldsymbol{\gamma}_{\mathbf{r r ^ { \prime }}}$ describes describes the irreversible interaction between the solid and the fluid. Note that these kernels are defined in (77) in terms of correlations involving the force density $\hat{\mathbf{F}}_{\mathbf{r}}^{s \rightarrow l}$ that the solid sphere exerts on the liquid molecules. These terms should be understood, therefore, as they are responsible for transmitting the irreversible forces that the solid sphere exerts on the fluid. force density $\hat{\mathbf{F}}_{\mathbf{r}}^{\mathrm{s} \rightarrow 1}(z)$ will be different from zero only for those points $\mathbf{r}$ that are near are near the solid sphere. This means that the transport kernels $\mathbf{G}_{\mathbf{r r}}, \mathbf{H}_{\mathbf{r r}^{\prime}}, \boldsymbol{\gamma}_{\mathbf{r r}^{\prime}}$ are highly highly localized near the atoms of the solid that interact with the fluid. Note that the concept of surface of the solid does not enter this theory. There is no such a well-defined surface in microscopic terms. However, this surface can be defined operationally by looking precisely at the behaviour of the above transport kernels. Indeed, the force density $\hat{\mathbf{F}}_{\mathbf{r}}^{\mathrm{s} \rightarrow \mathrm{l}}(z)$ will be a fluctuating vector on the surface of the solid sphere. If the interaction between fluid and solid atoms is purely repulsive, this force will be most of the time directed outwards the surface of the solid sphere. At the same time, because the interaction between solid and fluid atoms is singular as their separation vanishes, we expect that $\hat{\mathbf{F}}_{\mathbf{r}}^{\mathrm{s} \rightarrow 1}$ will diverge as $\mathbf{r}$ approaches the solid divergence will be reflected in divergences of the transport kernels as $\mathbf{r}$ or $\mathbf{r}^{\prime}$ approach the boundary of the solid. In a the solid. In a similar way, the stress tensor $\hat{\sigma}_{\mathbf{r}}$ that depends only on fluid atom coordinates will be non-zero only outside the solid sphere because the interaction with the solid sphere refrains to have liquid molecules inside the solid sphere (more on this later). Therefore, the surface of the solid may be characterized by the singularity of the transport coefficients $\mathbf{H}_{\mathbf{r} \mathbf{r}^{\prime}}, \mathbf{G}_{\mathbf{r r}^{\prime}}, \boldsymbol{\gamma}_{\mathbf{r r}^{\prime}}$ and the vanishing of $\boldsymbol{\eta}_{\mathbf{r} \mathbf{r}^{\prime}}$.

The force density and the stress tensor are assumed to vary in time in a time scale much shorter than the typical time scale in which the mass and momentum density of the solvent and the position and momentum of the solid sphere particle appreciably change. This separation of time scales is at the core of the Markovian form of the evolution of the CG dynamics in the projection operator technique. Whether the selected relevant variables do actually comply with this separation of time scales can only be assessed from the validity of the predictions of the resulting theory as compared with actual simulations or experiments. Note, however, that for slowly varying flow configurations, as those attained in steady states, the Markovian approximation should be reasonably fulfilled. 


\section{A. Conserved quantities, $\boldsymbol{H}$-theorem, and the equilibrium state}

Equations (78) are the equations of hydrodynamics in the presence of a solid spherical particle. We stress that the above equations conserve total mass and momentum, given by

$$
\begin{aligned}
M_{T} & =M^{\prime}+\int d \mathbf{r} \rho(\mathbf{r}), \\
\mathbf{P}_{T} & =\mathbf{P}+\int d \mathbf{r g}(\mathbf{r}) .
\end{aligned}
$$

Mass conservation follows immediately from the continuity equation. Momentum conservation is a consequence of the invariance under translations of the free energy functional expressed in Eq. (55) that ensures that the reversible part of the dynamics conserves momentum. The irreversible part conserves also total momentum because the momentum lost by the fluid is gained by the solid sphere.

In addition, the function $\mathcal{H}$ evolves in time in a strictly decreasing way, that is,

$$
\frac{d \mathcal{H}}{d t}(t) \leq 0
$$

where the equality sign occurs when the system has reached its equilibrium state. This is because, while the reversible part of the dynamics conserves $\mathcal{H}$, the irreversible part of the dynamics fulfills (80), due to the positive semidefinite character of the friction matrix.

As a consequence, the equilibrium state of Eqs. (78) is the one that minimizes $\mathcal{H}$ subject to the constraints of giving the actual values of the total mass and momentum. In order to obtain the equilibrium values of the relevant variables, one should minimize the following functional without constraints:

$$
\mathcal{H}-\mu_{0} \int d \mathbf{r} \rho(\mathbf{r})-\mathbf{V}_{0} \cdot\left[\int d \mathbf{r g}(\mathbf{r})+\mathbf{P}\right],
$$

where $\mu_{0}$ and $\mathbf{V}_{0}$ are the corresponding Lagrange multipliers. Setting to zero the derivatives of the above functional gives

$$
\begin{array}{rlrl}
\frac{\mathbf{v}^{2}(\mathbf{r})}{2}+\frac{\delta \mathcal{F}}{\delta \rho(\mathbf{r})}[\rho, \mathbf{R}]-\mu_{0} & =0, & & \frac{\partial \mathcal{F}}{\partial \mathbf{R}}[\rho, \mathbf{R}]=0, \\
\mathbf{v}(\mathbf{r})-\mathbf{V}_{0}=0, & \mathbf{V}-\mathbf{V}_{0}=0 .
\end{array}
$$

This means that the state of equilibrium has a constant velocity field equal to the velocity of the solid particle and, without losing generality, we may take $\mathbf{V}_{0}=0$. We then have the following two coupled equations for the equilibrium value that take the density field and the position of the sphere

$$
\frac{\delta \mathcal{F}}{\delta \rho(\mathbf{r})}[\rho, \mathbf{R}]=\mu_{0}, \quad \frac{\partial \mathcal{F}}{\partial \mathbf{R}}[\rho, \mathbf{R}]=0 .
$$

From Eqs. (49), the first Eq. (83) simply states that the equilibrium state has a constant value of the chemical potential $\mu(\mathbf{r})=\mu_{0}$, while the second equation states that the total force on the solid sphere vanishes at equilibrium. This set of two coupled equations should be understood as a set of nonlinear equations for the equilibrium density field $\rho(\mathbf{r})$ and the equilibrium value of the center of mass position $\mathbf{R}$ of the sphere. Translational invariance of the system implies that if $\mathbf{R}, \rho(\mathbf{r})$ is a solution of (83), then $\mathbf{R}+\mathbf{u}, \rho(\mathbf{r}+\mathbf{u})$ is also a solution. Therefore, without loss of generality, we may choose the origin of coordinates at the center of the sphere and $\mathbf{R}=0$. Then the equilibrium density field is the solution of the first equation. Note that for realistic models of the free energy functional, the density field will display oscillations at short length scales due to the packing of the fluid near the solid sphere.

In the present theory, the equilibrium profile is the one that is reached at long times due to the evolution of the hydrodynamic equations. In this subsection, we have shown how the theory presented contains the usual prescription to obtain the equilibrium density profile in DFT by minimizing the free energy functional.

\section{B. Approximating the relevant ensemble averages and projected currents}

It should be remarked that the theory presented is valid, as the H-theorem clearly testifies, for the decaying dynamics towards the equilibrium state in isolated systems. It is, therefore, a theory that describes, given initial non-equilibrium values for the averages of the CG variables, the subsequent average evolution towards equilibrium. One situation in which we will be interested is when the solid particle is very large and massive. In these situations, we may assume that the spherical particle has initial (average) position $\mathbf{R}_{0}$ and initial (average) momentum $\mathbf{P}_{0}=0$ and that, for all the decay evolution of the hydrodynamic fields, these values do not change appreciably because the forces that the fluid exerts on the particle during its evolution are not sufficiently strong to modify these variables substantially.

In principle, the relevant ensemble depends (in a functional way) on the averages of all the relevant variables of the system. As this is unduly complicated, we take the approximation in which the transport kernels are evaluated for the equilibrium values of the relevant variables. In doing this, we assume that for any other value of the average value of the relevant variables obtained in the course of the dynamics, the transport kernels do not change appreciably. The equilibrium values of the average relevant variables have been obtained in Eqs. (82) and (83) and are characterized by the following conjugate variables [see Eqs. (42) and (49)]:

$$
\begin{aligned}
\mu(\mathbf{r}, \mathbf{R}) & =\mu_{0}, \\
\lambda_{g}(\mathbf{r}) & =0, \\
\lambda_{P} & =0, \\
\lambda_{R} & =0 .
\end{aligned}
$$

Substitution of these conjugate variables into the relevant ensemble (35) shows that the relevant ensemble becomes just the equilibrium ensemble. Of course an equilibrium average of the microscopic system is one in which the sampling of the microscopic state involves states where the solid sphere may be located in any position of the physical space, due to Brownian motion. When the solid particle is infinitely massive, the position of the center of mass will not change appreciably in the time scale at which the solvent becomes equilibrated. That is, for a very massive sphere, we expect that the instantaneous values of their centers of mass $\hat{\mathbf{R}}$ are always very similar to their average values. For massive solid objects and, in particular, planar walls, we should understand the averages over the equilibrium ensemble as conditional averages where the 
solid object is fixed in space. These equilibrium averages can be, therefore, sampled through the ergodic hypothesis, as time averages over long MD simulations in which the solid remains fixed.

The form of the projected currents is computed explicitly in Appendix B.

When we approximate the relevant ensemble with an equilibrium ensemble in the Green-Kubo transport coefficients, they become state independent. In this particular case, we may justify Onsager's reciprocity for the transport coefficients. As it is well-known, if two phase space variables $\hat{A}(z), \hat{B}(z)$ transform under time reversal with the same parity, the property of microscopic reversibility together with the stationarity of the equilibrium ensemble implies

$$
\langle\hat{A}(t) B\rangle^{\mathrm{eq}}=\langle A \hat{B}(t)\rangle^{\mathrm{eq}} .
$$

The microscopic reversibility property reflects into the following property of correlations:

$$
\left\langle\left(\mathcal{Q} \mathbf{F}_{\mathbf{r}}(t)\right)\left(\mathcal{Q} \sigma_{\mathbf{r}^{\prime}}\right)\right\rangle^{\mathrm{eq}}=\left\langle\mathcal{Q} \sigma_{\mathbf{r}^{\prime}}(t) \mathcal{Q} \mathbf{F}_{\mathbf{r}}\right\rangle^{\mathrm{eq}} .
$$

This results in the following symmetry properties (Onsager relations) for the transport kernels:

$$
\begin{aligned}
\eta_{\mathbf{r r}}^{\alpha \beta \alpha^{\prime} \beta^{\prime}} & =\eta_{\mathbf{r}^{\prime} \mathbf{r}}^{\alpha^{\prime} \beta^{\prime} \alpha \beta}, \\
\mathbf{H}_{\mathbf{r} \mathbf{r}^{\prime}}^{\alpha \beta \alpha^{\prime}} & =\mathbf{g}_{\mathbf{r}^{\prime} \mathbf{r}}^{\alpha^{\prime} \alpha \beta}, \\
\gamma_{\mathbf{r} \mathbf{r}^{\prime}}^{\alpha \alpha^{\prime}} & =\gamma_{\mathbf{r}^{\prime} \mathbf{r}}^{\alpha^{\prime} \alpha} .
\end{aligned}
$$

\section{The solid made of frozen atoms}

In many simulation studies, solids in contact with fluid systems are modeled as made of atoms that are fixed in space. In this case, the atoms of the solid do not evolve according to Hamilton's equations and the theory presented does not apply. In fact, the atoms of the solid need to be understood as simple centers of force that generate an external field to be imposed on the fluid. The theory that emerges in this case is very different from the one that we have presented so far. A sketch of the derivation of the theory for fixed solid atoms is presented in what follows. The microstate of the system is given by the coordinates and positions of the fluid atoms. The coordinates $q^{\prime}$ of the solid atoms are no longer part of the microscopic state of the system but rather parameters in the Hamiltonian. Instead of the Hamiltonian (21), we have now

$$
H(z)=\sum_{i}^{N} \frac{p_{i}^{2}}{2 m_{i}}+V^{l}(q)+V^{l s}\left(q, q^{\prime}\right),
$$

where the coordinates of the solid atoms $q^{\prime}$ are fixed parameters of the Hamiltonian. The relevant variables are only the hydrodynamic variables of the solvent. We may follow all the steps that we have taken in Sec. V for the present case. The most important observation is that the microscopic force that the solid exerts on the fluid depends on the microscopic state $z$ of the system through the solvent density, which is a relevant variable. Indeed,

$$
\begin{aligned}
\hat{\mathbf{F}}_{\mathbf{r}}^{\mathrm{s} \rightarrow \mathrm{l}}=\sum_{i i^{\prime}}^{N N^{\prime}} \hat{\mathbf{F}}_{i i^{\prime}} \delta\left(\mathbf{r}-\mathbf{q}_{i}\right) & =\sum_{i i^{\prime}}^{N N^{\prime}} \mathbf{F}\left(\mathbf{r}-\mathbf{q}_{i^{\prime}}\right) \delta\left(\mathbf{r}-\mathbf{q}_{i}\right) \\
& =\hat{\mathbf{F}}(\mathbf{r}) \hat{\rho}_{\mathbf{r}}(z)
\end{aligned}
$$

where $\hat{\mathbf{F}}(\mathbf{r})$ is the force that all the frozen solid atoms exert on a fluid atom located at $\mathbf{r}$. This force does not evolve in time and is not a dynamic object at all, it is a given vector function that depends parametrically on the frozen positions of the solid atom. Because this force depends on the microscopic state through the density field, which is a relevant variable, the projected force $\mathcal{Q} \hat{\mathbf{F}}_{\mathbf{r}}^{\mathrm{s} \rightarrow 1}=0$ vanishes exactly. As a consequence, for this type of walls made offrozen atoms, the irreversible surface force $\mathcal{S}(\mathbf{r})$ vanishes identically because all the transport kernels involving the projected force vanish. This gives a strong indication about the physical origin of the irreversible surface force in the present level of description. It is due entirely to the eliminated internal degrees of freedom of the solid that are coarse-grained in the description, in terms of the center of mass of the solid sphere. While we have assumed that elastic degrees of freedom for the solid are very fast relaxing degrees of freedom towards equilibrium, it is precisely the unaccounted solid degrees of freedom responsible for the originators of the irreversible surface forces on the solvent. This point is subtle though. As we will see in a subsequent publication, a slightly different level of description, one in which the fluid is coarsegrained not with the local mass $\hat{\rho}_{\mathbf{r}}$ and momentum $\hat{\mathbf{g}}_{\mathbf{r}}$ densities at a given point of space, but rather as the mass $\hat{\rho}_{\mu}$ and momentum $\hat{\mathbf{g}}_{\mu}$ densities of fluid slabs labeled with index $\mu$ (suitable for planar geometries), produces irreversible forces even in the case that the solid degrees of freedom are frozen. This is due to the fact that in the later level of description, which is coarser than the former, the loss of information due to the use of fluid slabs is sufficient for the appearance of the irreversible forces. This is a nice example that illustrates a very general concept: Dissipation is not an intrinsic property of the system, but rather it is a function of the level of description used.

\section{DISCUSSION}

A crucial assumption in the method of projection operators is that the relevant variables should be slow in order to have a Markovian, memoryless equation. Equations with memory are indeed much more difficult to deal with than memoryless equations. In the former case, one needs to know the specific form of the memory kernel function instead of just one single number, the transport coefficient, which in the present theory is, in fact, a non-local function of space. Equations (78) are nonlocal in space because (1) the free energy functional depends in general in a non-local way on the density field in order to account for the ordering of a fluid near a wall. And (2), the irreversible part of the dynamics involves transport kernels that are non-local. Note that we assume non-locality in space but locality in time (no memory), and this may not be entirely consistent. However, memory kernels are typically uncomputable from MD, as they involve the projected dynamics instead of the real dynamics. The usual approximation in which the projected dynamics is substituted with the real, Hamiltonian, dynamics is only justified when, precisely, one has separation of time scales (i.e., when the Markovian dynamics holds). We take the pragmatic point of view in which we give credit to the Markovian approximation but retain spatially non-local hydrodynamic equations by hoping that non-Markovian effects will not dominate the problem. In particular, we believe that for 
flows that vary slowly in time, the Markov approximation will hold. This excludes, perhaps, shocks or other strongly varying flows.

The theory presented in Sec. II is valid for isolated systems that decay towards the unique equilibrium state, abiding to the second law of thermodynamics, and it does not allow to treat forced situations where there are external forces applied either to the fluid or to the solid. In this respect, no steady states can be described by the theory as it stands. However, the generalization of the framework to include external forcing is not difficult and is sketched in what follows by following Grabert's textbook. ${ }^{21}$ For sufficiently small external forcings, the equations of motion (i.e., the transport equations and free energy function) are the same as the no forced situations, with the external forcing appearing in two places. On the one hand, in the fluid momentum equation, a term $\rho(\mathbf{r}, t) \mathbf{F}^{\text {ext }}(\mathbf{r}, t)$ appears, where $\mathbf{F}^{\text {ext }}(\mathbf{r}, t)$ is any external force field acting on a molecule of fluid that happens to be at the point $\mathbf{r}$. On the other hand, in the particle momentum equation, an additional force term $\mathbf{F}(\mathbf{R})$ appears, describing any external force acting on the particle.

The theory presented is isothermal. We have not considered in the description the energy density field of the fluid nor the internal energy of the solid sphere. Instead, only the total energy has been considered as a relevant variable and local transport of energy is not described by the present theory. Of course, these local energy variables can be introduced in the theory, at the expense of additional equations and terms. In a future publication, we will address the non-isothermal theory.

A word is in order about the mathematical nature of the integro-differential equations. From a mathematical point of view, these equations require the specification of (mathematical) boundary conditions in order to have a unique solution. What are the suitable boundary conditions to be specified for Eqs. (78)? In the present situation, where only transient dynamics towards equilibrium can be investigated, these boundary conditions appear as an initial value problem. In addition, note that we have not included any external confining potential that would model the confinement of the fluid in a container. Only a sphere in an infinite fluid (or with periodic boundary conditions) has been considered. It is straightforward to include an external potential, though. These confining potentials are such that, by definition, they leave the fluid in a closed region of space, where outside the region there are no atoms of the fluid. In order to do so, these confining potentials need to be singular. This implies that outside the container region both the mass density and momentum densities of the fluid are zero. Note that outside the container region, both the force density $\hat{\mathbf{F}}_{\mathbf{r}}^{s \rightarrow 1}(z)$ and the fluid stress tensor $\hat{\boldsymbol{\sigma}}_{\mathbf{r}}(z)$ vanish because there are no fluid particles outside. This means that the transport kernels vanish if $\mathbf{r}$ or $\mathbf{r}^{\prime}$ are outside the container. This should tame the potentially singular presence of factors of the inverse of the density appearing in the velocity field $\mathbf{v}(\mathbf{r})$ $=\mathbf{g}(\mathbf{r}) / \rho(\mathbf{r})$. The presence of the sphere produces forces that are highly localized in a narrow region around the solid particle. From a thermodynamic point of view, the issue of boundary conditions as emerging from "physics" rather than "numerical analysis" has also been considered in the past. ${ }^{12,42-44} \mathrm{In}$ a fully macroscopic phenomenological theory, the effect of the irreversible surface force is taken into account through boundary conditions applied to the fluid equations. We will discuss in a future publication the emergence of boundary conditions in a macroscopic theory. There, we will consider the conditions under which the localized irreversible surface forces produce actual boundary conditions for the hydrodynamic equations.

A general comment on the status of the present theory is in order. This theory is the natural generalization of equilibrium density functional theory for simple fluids, when these fluids are in motion and, therefore, out of equilibrium. It allows, in principle, to study effects like how the structuring of the fluid near a wall is modified by the flow field. While it is a coarse-grained theory where the relevant variables are the hydrodynamic fields, it captures the structure of the fluid at atomic nanoscales. The value of the present theory lies in that it provides the structure of the equations. For example, as compared with previous work, the present theory gives a neat definition of all the irreversible forces in a fluid that arise due to the interaction with solids. In particular, the distinction of the forces on the fluid due to the fluid itself and due to the solid reflects into the final structure of the irreversible forces that involve both microscopically defined slip coefficients (due to fluid-solid interactions) as well as viscosity kernels (due to the fluid-fluid interactions). The form of these forces is such that not only friction forces that depend on the velocity difference between the fluid and the solid appear but also forces that depend on the gradients of the velocity field near the solid are present.

All these features are important assets of the theory. Unfortunately, in order to make the theory predictive, it is necessary to fill in some important information. The first input element is the free energy functional (56) involving the standard equilibrium free energy functional $\mathcal{F}_{0}[\rho]$. One can benefit from the vast amount of work in the literature addressing the construction of very realistic models for the free energy functional. The second piece of information that is required is the dissipative matrix that contains the non-local transport kernels, which are tensorial in nature. This means that in order to make dynamic predictions with this theory, we have to know in advance 50 different functions (tensor elements after sorting out the corresponding symmetries of the stress tensor and Onsager reciprocity) of the six variables $\mathbf{r}, \mathbf{r}^{\prime}$. While in principle, the Green-Kubo expressions would allow for the explicit calculation through MD simulations, it is clearly not a feasible program. In order to make any advance in the prediction of transport at the nanoscale, it is necessary to make some approximations in order to reduce the large number of transport kernels. In future work, we consider situations in which symmetries of the solid surface and fluid flow can be exploited. In those situations, the number of Green-Kubo expressions is dramatically reduced and can be computed explicitly.

The present theory is concerned with the evolution of the averages of the relevant variables, in this case, the hydrodynamic fields and the momentum and position of the solid sphere. This precludes the study of Brownian motion of the particle. The average position of a Brownian particle may be zero even though the variance of the position increases in time. Brownian motion is captured by the probability 
distribution of the position and momenta of the solid particle. We are not interested in the present paper in the Brownian motion of nanoparticles and the subtle role of hydrodynamic fluctuations. We have addressed these issues in a separate publication. ${ }^{45}$ In the present paper, we focus on the time-dependent ensemble averages of the hydrodynamic fields and the position and momentum of the particle because our interest in describing the unifying framework in which density functional theory and hydrodynamics coexists. The fact that we use a spherical solid particle is just a recourse to deal with momentum conservation, but we have in mind that the particle will be very large and massive (in fact it will be used eventually to represent a wall) for which Brownian motion plays no role.

\section{ACKNOWLEDGMENTS}

Useful discussions with Aleks Donev are very appreciated. Diego Camargo is grateful to COLCIENCIAS-Colombia for supporting this work through Doctoral Scholarship program. This research is supported by the Ministerio de Economía y Competitividad of Spain (MINECO) under Grant Nos. FIS2013-47350-C5-1-R and FIS2013-47350-C5-3-R.

\section{APPENDIX A: FORCES}

In this appendix, we summarize the different forces and force densities introduced so far and present some results concerning its averages with the relevant ensemble. The force densities that the fluid or the solid exerts on a fluid molecule located at the point $\mathbf{r}$ are introduced in (27),

$$
\begin{gathered}
\hat{\mathbf{F}}_{\mathbf{r}}^{l \rightarrow 1}(z) \equiv \sum_{i j}^{N N} \hat{\mathbf{F}}_{i j} \delta\left(\mathbf{r}-\mathbf{q}_{i}\right), \\
\hat{\mathbf{F}}_{\mathbf{r}}^{\mathrm{s} \rightarrow 1}(z) \equiv \sum_{i j^{\prime}}^{N N^{\prime}} \hat{\mathbf{F}}_{i j^{\prime}} \delta\left(\mathbf{r}-\mathbf{q}_{i}\right),
\end{gathered}
$$

where $\hat{\mathbf{F}}_{i j^{\prime}}$ is the force that atom $j^{\prime}$ of the solid exerts on atom $i$ of the liquid. That is, $\hat{\mathbf{F}}_{\mathbf{r}}^{l \rightarrow l}(z)$ is the force density that the liquid exerts on the liquid molecules that are around the point $\mathbf{r}$, while $\hat{\mathbf{F}}_{\mathbf{r}}^{\mathrm{s} \rightarrow \mathrm{l}}(z)$ is the force density that the solid exerts on the liquid at the point $\mathbf{r}$.

The total force density on the fluid is

$$
\hat{\mathbf{F}}_{\mathbf{r}}^{l}=\hat{\mathbf{F}}_{\mathbf{r}}^{l \rightarrow 1}+\hat{\mathbf{F}}_{\mathbf{r}}^{s \rightarrow 1}=\sum_{j}^{N}-\frac{\partial U}{\partial \mathbf{q}_{j}} \delta\left(\mathbf{r}-\mathbf{q}_{j}\right) .
$$

The total force on the fluid and on the solid is

$$
\begin{aligned}
\hat{\mathbf{F}}^{l} & =\int d \mathbf{r} \hat{\mathbf{F}}_{\mathbf{r}}^{l}, \\
\hat{\mathbf{F}}^{s} & =-\hat{\mathbf{F}}^{l},
\end{aligned}
$$

where we have used that the total force

$$
\hat{\mathbf{F}}=-\sum_{i}^{N} \frac{\partial U}{\partial \mathbf{q}_{i}}-\sum_{i^{\prime}}^{N^{\prime}} \frac{\partial U}{\partial \mathbf{q}_{i^{\prime}}}=\hat{\mathbf{F}}^{l}+\hat{\mathbf{F}}^{s}
$$

vanishes because the potentials are translational invariant (and therefore, Newton's third law holds).

Note that we have the result

$$
\hat{\mathbf{F}}^{s}=-\int d \mathbf{r} \hat{\mathbf{F}}_{\mathbf{r}}^{s \rightarrow 1}
$$

Now, let us consider the average of the total force density on the liquid, Eq. (26)

$$
\begin{aligned}
\mathbf{F}_{\mathbf{r}}^{l} \equiv & \operatorname{Tr}\left[\bar{\rho}_{t} \hat{\mathbf{F}}_{\mathbf{r}}^{l}\right]=\left\langle\hat{\mathbf{F}}_{\mathbf{r}}^{l}\right\rangle^{\mu, \lambda_{R}} \\
= & \frac{1}{\Xi\left[\mu, \lambda_{R}\right]} \sum_{N=0}^{\infty} \frac{1}{N !} \int \frac{d q}{\Lambda^{3 N}} \frac{d q^{\prime}}{\Lambda^{3 N^{\prime}}}\left[\sum_{j}^{N}-\frac{\partial U}{\partial \mathbf{q}_{j}} \delta\left(\mathbf{r}-\mathbf{q}_{j}\right)\right] e^{-\beta U} \\
& \times \exp \left\{-\beta\left(\lambda_{R} \cdot \hat{\mathbf{R}}-\sum_{i=1}^{N} m \mu\left(\mathbf{q}_{i}\right)\right)\right\},
\end{aligned}
$$

where we have used the definition of the average with respect to the relevant ensemble, after performing the momentum integrals. Next, we realize that the derivative of the Boltzmann factor is the potential times the Boltzmann factor itself, that is,

$$
\begin{aligned}
\mathbf{F}_{\mathbf{r}}^{l}= & \frac{1}{\Xi\left[\mu, \lambda_{R}\right]} \sum_{N=0}^{\infty} \frac{1}{N !} \int \frac{d q}{\Lambda^{3 N}} \frac{d q^{\prime}}{\Lambda^{3 N^{\prime}}} \\
& \times \exp \left\{-\beta\left(\lambda_{R} \cdot \hat{\mathbf{R}}-\sum_{i=1}^{N} m \mu\left(\mathbf{r}_{i}\right)\right)\right\} \\
& \times k_{B} T \sum_{j}^{N} \delta\left(\mathbf{r}-\mathbf{q}_{j}\right) \frac{\partial}{\partial \mathbf{q}_{j}} e^{-\beta U} .
\end{aligned}
$$

Integrate by parts to obtain

$$
\begin{aligned}
\mathbf{F}_{\mathbf{r}}^{l}= & \frac{1}{\Xi\left[\mu, \lambda_{R}\right]} \sum_{N=0}^{\infty} \frac{1}{N !} \int \frac{d q}{\Lambda^{3 N}} \frac{d q^{\prime}}{\Lambda^{3 N^{\prime}}} e^{-\beta U} k_{B} T \sum_{j}^{N}-\frac{\partial}{\partial \mathbf{q}_{j}}\left[\delta\left(\mathbf{r}-\mathbf{q}_{j}\right) \exp \left\{-\beta\left(\lambda_{R} \cdot \hat{\mathbf{R}}-\sum_{i=1}^{N} m \mu\left(\mathbf{q}_{i}\right)\right)\right\}\right] \\
= & \frac{1}{\Xi\left[\mu, \lambda_{R}\right]} \sum_{N=0}^{\infty} \frac{1}{N !} \int \frac{d q}{\Lambda^{3 N}} \frac{d q^{\prime}}{\Lambda^{3 N^{\prime}}} e^{-\beta U} \sum_{j}^{N} \exp \left\{-\beta\left(\lambda_{R} \cdot \hat{\mathbf{R}}-\sum_{i \neq j}^{N} m \mu\left(\mathbf{q}_{j}\right)\right)\right\} \\
& \times k_{B} T\left[-\frac{\partial}{\partial \mathbf{q}_{j}} \delta\left(\mathbf{r}-\mathbf{q}_{j}\right) \exp \left\{\beta m \mu\left(\mathbf{q}_{j}\right)\right\}\right] \\
= & \frac{k_{B} T}{m} \boldsymbol{\nabla} \rho(\mathbf{r})-\rho(\mathbf{r}) \boldsymbol{\nabla} \mu(\mathbf{r}) .
\end{aligned}
$$

By following identical steps, we may compute the average of the total force on the solid $\mathbf{F}^{s}$ and obtain 


$$
\begin{aligned}
\mathbf{F}^{s} & =\operatorname{Tr}\left[\bar{\rho}_{t} \hat{\mathbf{F}}^{s}\right]=\left\langle\hat{\mathbf{F}}^{s}\right\rangle^{\mu, \lambda_{R}} \\
& =\frac{1}{\Xi\left[\mu, \lambda_{R}\right]} \sum_{N=0}^{\infty} \frac{1}{N !} \int \frac{d q}{\Lambda^{3 N}} \frac{d q^{\prime}}{\Lambda^{3 N^{\prime}}}\left[-\sum_{i^{\prime}}^{N^{\prime}} \frac{\partial U}{\partial \mathbf{q}_{i^{\prime}}}\right] e^{-\beta U} \exp \left\{-\beta\left(\sum_{i=1}^{N} m \mu\left(\mathbf{r}_{i}\right)+\lambda_{R} \cdot \hat{\mathbf{R}}\right)\right\} \\
& =\frac{1}{\Xi\left[\mu, \lambda_{R}\right]} \sum_{N=0}^{\infty} \frac{1}{N !} \int \frac{d q}{\Lambda^{3 N}} \frac{d q^{\prime}}{\Lambda^{3 N^{\prime}}} \exp \left\{-\beta\left(\sum_{i=1}^{N} m \mu\left(\mathbf{r}_{i}\right)+\lambda_{R} \cdot \hat{\mathbf{R}}\right)\right\} k_{B} T\left[\sum_{i^{\prime}}^{N^{\prime}} \frac{\partial}{\partial \mathbf{q}_{i^{\prime}}}\right] e^{-\beta U} \\
& =\frac{1}{\Xi\left[\mu, \lambda_{R}\right]} \sum_{N=0}^{\infty} \frac{1}{N !} \int \frac{d q}{\Lambda^{3 N}} \frac{d q^{\prime}}{\Lambda^{3 N^{\prime}}} e^{-\beta U} k_{B} T\left[-\sum_{i^{\prime}}^{N^{\prime}} \frac{\partial}{\partial \mathbf{q}_{i^{\prime}}}\right] \exp \left\{-\beta\left(\sum_{i=1}^{N} m \mu\left(\mathbf{r}_{i}\right)+\lambda_{R} \cdot \hat{\mathbf{R}}\right)\right\} \\
& =\frac{1}{\Xi\left[\mu, \lambda_{R}\right]} \sum_{N=0}^{\infty} \frac{1}{N !} \int \frac{d q}{\Lambda^{3 N}} \frac{d q^{\prime}}{\Lambda^{3 N^{\prime}}} e^{-\beta U} \exp \left\{-\beta\left(\sum_{i=1}^{N} m \mu\left(\mathbf{r}_{i}\right)+m \mu\left(\mathbf{r}_{i}\right)+\lambda_{R} \cdot \hat{\mathbf{R}}\right)\right\}\left[\sum_{i^{\prime}}^{N^{\prime}} \frac{\partial}{\partial \mathbf{q}_{i^{\prime}}}\right] \lambda_{R} \cdot \hat{\mathbf{R}} \\
& =\lambda_{R} .
\end{aligned}
$$

This identity allows one to interpret physically the Lagrange multiplier $\lambda_{R}$ as the force on the solid sphere. By using (49), we obtain that the total force on the solid sphere is due to the gradient of the free energy functional

$$
\mathbf{F}^{s}=-\frac{\partial \mathcal{F}}{\partial \mathbf{R}}[\rho, \mathbf{R}]
$$

\section{APPENDIX B: THE PROJECTED CURRENTS}

In this appendix, we consider the explicit form of the projected currents $\mathcal{Q}_{t} i L \hat{A}$ for the present selection of relevant variables. The projector defined in (17) gives rise to the following two projected currents $\mathcal{Q}_{t} \hat{\mathbf{F}}_{\mathbf{r}}^{\text {s } \rightarrow 1}, \mathcal{Q}_{t} \hat{\sigma}_{\mathbf{r}}$ given explicitly by

$$
\begin{aligned}
\mathcal{Q}_{t} \hat{\mathbf{F}}_{\mathbf{r}}^{s \rightarrow 1}= & \hat{\mathbf{F}}_{\mathbf{r}}^{s \rightarrow 1}-\operatorname{Tr}\left[\bar{\rho}_{t} \hat{\mathbf{F}}_{\mathbf{r}}^{s \rightarrow 1}\right]-(\hat{\mathbf{R}}-\mathbf{R}(t)) \cdot \frac{\partial}{\partial \mathbf{R}(t)} \operatorname{Tr}\left[\bar{\rho}_{t} \hat{\mathbf{F}}_{\mathbf{r}}^{s \rightarrow 1}\right]-(\hat{\mathbf{P}}-\mathbf{P}(t)) \cdot \frac{\partial}{\partial \mathbf{P}(t)} \operatorname{Tr}\left[\bar{\rho}_{t} \hat{\mathbf{F}}_{\mathbf{r}}^{s \rightarrow 1}\right] \\
& -\int d \mathbf{r}^{\prime}\left(\hat{\rho}_{\mathbf{r}^{\prime}}-\rho\left(\mathbf{r}^{\prime}, t\right)\right) \frac{\delta}{\delta \rho\left(\mathbf{r}^{\prime}, t\right)} \operatorname{Tr}\left[\bar{\rho}_{t^{\prime}} \hat{\mathbf{F}}_{\mathbf{r}}^{s \rightarrow 1}\right]-\int d \mathbf{r}^{\prime}\left(\hat{\mathbf{g}}_{\mathbf{r}^{\prime}}-\mathbf{g}\left(\mathbf{r}^{\prime}, t\right)\right) \cdot \frac{\delta}{\delta \mathbf{g}\left(\mathbf{r}^{\prime}, t\right)} \operatorname{Tr}\left[\bar{\rho}_{t^{\prime}} \hat{\mathbf{F}}_{\mathbf{r}}^{s \rightarrow 1}\right]
\end{aligned}
$$

and

$$
\begin{aligned}
\mathcal{Q}_{t} \hat{\boldsymbol{\sigma}}_{\mathbf{r}}= & \hat{\boldsymbol{\sigma}}_{\mathbf{r}}-\operatorname{Tr}\left[\bar{\rho}_{t} \hat{\boldsymbol{\sigma}}_{\mathbf{r}}\right]-(\hat{\mathbf{R}}-\mathbf{R}(t)) \cdot \frac{\partial}{\partial \mathbf{R}(t)} \operatorname{Tr}\left[\bar{\rho}_{t} \hat{\boldsymbol{\sigma}}_{\mathbf{r}}\right]-(\hat{\mathbf{P}}-\mathbf{P}(t)) \cdot \frac{\partial}{\partial \mathbf{P}(t)} \operatorname{Tr}\left[\bar{\rho}_{t} \hat{\boldsymbol{\sigma}}_{\mathbf{r}}\right] \\
& -\int d \mathbf{r}^{\prime}\left(\hat{\rho}_{\mathbf{r}^{\prime}}-\rho\left(\mathbf{r}^{\prime}, t\right)\right) \frac{\delta}{\delta \rho\left(\mathbf{r}^{\prime}, t\right)} \operatorname{Tr}\left[\bar{\rho}_{t^{\prime}} \hat{\boldsymbol{\sigma}}_{\mathbf{r}}\right]-\int d \mathbf{r}^{\prime}\left(\hat{\mathbf{g}}_{\mathbf{r}^{\prime}}-\mathbf{g}\left(\mathbf{r}^{\prime}, t\right)\right) \cdot \frac{\delta}{\delta \mathbf{g}\left(\mathbf{r}^{\prime}, t\right)} \operatorname{Tr}\left[\bar{\rho}_{t^{\prime}} \hat{\boldsymbol{\sigma}}_{\mathbf{r}}\right]
\end{aligned}
$$

Under the assumption that the solid particle is sufficiently large, the actual values $\hat{\mathbf{R}}, \hat{\mathbf{P}}$ of the microscopic functions will not differ too much from its average values, and the corresponding terms in (B1) and (B2) may be neglected. The projected currents become

$$
\begin{aligned}
\mathcal{Q}_{t} \hat{\mathbf{F}}_{\mathbf{r}}^{s \rightarrow 1}= & \hat{\mathbf{F}}_{\mathbf{r}}^{\mathrm{s} \rightarrow 1}-\operatorname{Tr}\left[\bar{\rho}_{t} \hat{\mathbf{F}}_{\mathbf{r}}^{\mathrm{r} \rightarrow 1}\right] \\
& -\int d \mathbf{r}^{\prime}\left(\hat{\rho}_{\mathbf{r}^{\prime}}-\rho\left(\mathbf{r}^{\prime}, t\right)\right) \frac{\delta}{\delta \rho\left(\mathbf{r}^{\prime}, t\right)} \operatorname{Tr}\left[\bar{\rho}_{t^{\prime}} \hat{\mathbf{F}}_{\mathbf{r}}^{s \rightarrow 1}\right] \\
& -\int d \mathbf{r}^{\prime}\left(\hat{\mathbf{g}}_{\mathbf{r}^{\prime}}-\mathbf{g}\left(\mathbf{r}^{\prime}, t\right)\right) \cdot \frac{\delta}{\delta \mathbf{g}\left(\mathbf{r}^{\prime}, t\right)} \operatorname{Tr}\left[\bar{\rho}_{t^{\prime}} \hat{\mathbf{F}}_{\mathbf{r}}^{s \rightarrow 1}\right]
\end{aligned}
$$

and

$$
\begin{aligned}
\mathcal{Q}_{t} \hat{\boldsymbol{\sigma}}_{\mathbf{r}}= & \hat{\boldsymbol{\sigma}}_{\mathbf{r}}-\operatorname{Tr}\left[\bar{\rho}_{t} \hat{\boldsymbol{\sigma}}_{\mathbf{r}}\right] \\
& -\int d \mathbf{r}^{\prime}\left(\hat{\rho}_{\mathbf{r}^{\prime}}-\rho\left(\mathbf{r}^{\prime}, t\right)\right) \frac{\delta}{\delta \rho\left(\mathbf{r}^{\prime}, t\right)} \operatorname{Tr}\left[\bar{\rho}_{t^{\prime}} \hat{\boldsymbol{\sigma}}_{\mathbf{r}}\right] \\
& -\int d \mathbf{r}^{\prime}\left(\hat{\mathbf{g}}_{\mathbf{r}^{\prime}}-\mathbf{g}\left(\mathbf{r}^{\prime}, t\right)\right) \cdot \frac{\delta}{\delta \mathbf{g}\left(\mathbf{r}^{\prime}, t\right)} \operatorname{Tr}\left[\bar{\rho}_{t^{\prime}} \hat{\boldsymbol{\sigma}}_{\mathbf{r}}\right]
\end{aligned}
$$

Let us start with the projected current (B3) that requires the average with the relevant ensemble of the force density that the solid exerts on the fluid

$$
\operatorname{Tr}\left[\bar{\rho}_{t^{\prime}} \hat{\mathbf{F}}_{\mathbf{r}}^{\mathrm{s} \rightarrow 1}\right]=\operatorname{Tr}\left[\mathcal{G} \bar{\rho}_{t^{\prime}} \mathcal{G} \hat{\mathbf{F}}_{\mathbf{r}}^{\mathrm{s} \rightarrow 1}\right] .
$$

Because the force does not depend on velocities, the Galilean operator does nothing on it. Therefore, we need to compute

$$
\begin{aligned}
\operatorname{Tr}\left[\bar{\rho}_{t^{\prime}} \hat{\mathbf{F}}_{\mathbf{r}}^{\mathrm{s} \rightarrow 1}\right]= & \frac{1}{\Xi[\lambda(t)]} \operatorname{Tr}\left[\rho ^ { \mathrm { eq } } ( z ) \operatorname { e x p } \left\{\beta \int d \mathbf{r} \mu(\mathbf{r}) \hat{\rho}_{\mathbf{r}}(z)\right.\right. \\
& \left.\left.-\beta \lambda_{R}(t) \cdot \hat{\mathbf{R}}-\beta \lambda_{P}(t) \cdot \hat{\mathbf{P}}\right\} \hat{\mathbf{F}}_{\mathbf{r}}^{\mathrm{s} \rightarrow 1}\right],
\end{aligned}
$$

which does not depend on the momentum of the fluid (because none of the conjugate variables does). Note that the average of the force density that the solid exerts on the fluid does not depend on the momentum variable, and the last term in (B3) vanishes. We end up, therefore, with the following result: 


$$
\mathcal{Q}_{t} \hat{\mathbf{F}}_{\mathbf{r}}^{\mathrm{s} \rightarrow 1}=\hat{\mathbf{F}}_{\mathbf{r}}^{\mathrm{s} \rightarrow 1}-\operatorname{Tr}\left[\bar{\rho}_{t} \hat{\mathbf{F}}_{\mathbf{r}}^{\mathrm{s} \rightarrow 1}\right]-\int d \mathbf{r}^{\prime}\left(\hat{\rho}_{\mathbf{r}^{\prime}}-\rho\left(\mathbf{r}^{\prime}, t\right)\right) \frac{\delta}{\delta \rho\left(\mathbf{r}^{\prime}, t\right)} \operatorname{Tr}\left[\bar{\rho}_{t} \hat{\mathbf{F}}_{\mathbf{r}}^{\mathrm{s} \rightarrow 1}\right] .
$$

Note that the functional derivative of the relevant ensemble is

$$
\begin{aligned}
\frac{\delta}{\delta \rho\left(\mathbf{r}^{\prime}, t\right)} \bar{\rho}_{t} & =\frac{\delta}{\delta \rho\left(\mathbf{r}^{\prime}, t\right)} \frac{1}{\Xi[\lambda(t)]} \rho^{\mathrm{eq}}(z) \exp \left\{\beta \int d \mathbf{r} \mu(\mathbf{r}) \hat{\rho}_{\mathbf{r}}(z)-\beta \lambda_{R}(t) \cdot \hat{\mathbf{R}}-\beta \lambda_{P}(t) \cdot \hat{\mathbf{P}}\right\} \\
& =\bar{\rho}_{t}\left[\frac{\delta}{\delta \rho\left(\mathbf{r}^{\prime}, t\right)} \beta \rho\left(\mathbf{r}^{\prime}, t\right) \beta \int d \mathbf{r} \mu(\mathbf{r}) \hat{\rho}_{\mathbf{r}}(z)-\frac{\delta}{\delta \rho\left(\mathbf{r}^{\prime}, t\right)} \ln \Xi[\lambda(t)]\right] \\
& =\bar{\rho}_{t} \beta \int d \mathbf{r}^{\prime} \frac{\delta \mu\left(\mathbf{r}^{\prime \prime}\right)}{\delta \rho\left(\mathbf{r}^{\prime}, t\right)} \delta \hat{\rho}_{\mathbf{r}^{\prime \prime}}(z),
\end{aligned}
$$

where $\delta \hat{\rho}_{\mathbf{r}^{\prime \prime}}(z)=\hat{\rho}_{\mathbf{r}^{\prime \prime}}(z)-\rho\left(\mathbf{r}^{\prime \prime}, t\right)$. We have neglected terms that involve the functional derivative of $\lambda_{\mathbf{R}}$ and $\lambda_{\mathbf{P}}$ because they accompany fluctuations of $\mathbf{R}$ and $\mathbf{P}$ which are assumed to be negligible.

We need to evaluate the functional derivative of the chemical potential with respect to the number density field. This can be achieved by noting that

$$
\begin{aligned}
\frac{\delta}{\delta \mu(\mathbf{r})} \ln \Xi[\lambda] & =\beta \rho(\mathbf{r}), \\
\frac{\delta^{2}}{\delta \mu(\mathbf{r}) \delta \mu\left(\mathbf{r}^{\prime}\right)} \ln \Xi[\lambda] & =\beta^{2}\langle\delta \hat{\rho}(\mathbf{r}) \delta \hat{\rho}(\mathbf{r})\rangle,
\end{aligned}
$$

which both imply

$$
\frac{\delta \rho\left(\mathbf{r}^{\prime}\right)}{\delta \mu(\mathbf{r})}=\beta\left\langle\delta \hat{\rho}_{\mathbf{r}} \delta \hat{\rho}_{\mathbf{r}^{\prime}}\right\rangle
$$

The functional derivative appearing in (B8) is, therefore, the inverse of the density correlation matrix, that is,

$$
\frac{\delta \mu(\mathbf{r})}{\delta \rho\left(\mathbf{r}^{\prime}\right)}=\beta^{-1}\left\langle\delta \hat{\rho}_{\mathbf{r}} \delta \hat{\rho}_{\mathbf{r}^{\prime}}\right\rangle^{-1} .
$$

Therefore, the projected current (B7) is

$$
\begin{aligned}
\mathcal{Q}_{t} \hat{\mathbf{F}}_{\mathbf{r}}^{\mathrm{s} \rightarrow 1}= & \hat{\mathbf{F}}_{\mathbf{r}}^{\mathrm{s} \rightarrow 1}-\operatorname{Tr}\left[\bar{\rho}_{t} \hat{\mathbf{F}}_{\mathbf{r}}^{\mathrm{s} \rightarrow 1}\right]-\int d \mathbf{r}^{\prime} \\
& \times \int d \mathbf{r}^{\prime \prime}\left(\hat{\rho}_{\mathbf{r}^{\prime}}-\rho\left(\mathbf{r}^{\prime}, t\right)\right)\left\langle\delta \hat{\rho}_{\mathbf{r}^{\prime}} \delta \hat{\rho}_{\mathbf{r}^{\prime \prime}}\right\rangle^{-1}\left\langle\delta \hat{\rho}_{\mathbf{r}^{\prime}} \hat{\mathbf{F}}_{\mathbf{r}}^{\mathrm{s} \rightarrow 1}\right\rangle .
\end{aligned}
$$

Let us now move to the projected current $\mathcal{Q}_{t} \hat{\sigma}_{\mathbf{r}}$,

$$
\begin{aligned}
\mathcal{Q}_{t} \hat{\boldsymbol{\sigma}}_{\mathbf{r}}= & \hat{\boldsymbol{\sigma}}_{\mathbf{r}}-\operatorname{Tr}\left[\bar{\rho}_{t} \hat{\boldsymbol{\sigma}}_{\mathbf{r}}\right]-\int d \mathbf{r}^{\prime}\left(\hat{\rho}_{\mathbf{r}^{\prime}}-\rho\left(\mathbf{r}^{\prime}, t\right)\right) \frac{\delta}{\delta \rho\left(\mathbf{r}^{\prime}, t\right)} \operatorname{Tr}\left[\bar{\rho}_{t^{\prime}} \hat{\boldsymbol{\sigma}}_{\mathbf{r}}\right]-\int d \mathbf{r}^{\prime}\left(\hat{\mathbf{g}}_{\mathbf{r}^{\prime}}-\mathbf{g}\left(\mathbf{r}^{\prime}, t\right)\right) \frac{\delta}{\delta \mathbf{g}\left(\mathbf{r}^{\prime}, t\right)} \operatorname{Tr}\left[\bar{\rho}_{t^{\prime}} \hat{\boldsymbol{\sigma}}_{\mathbf{r}}\right] \\
= & \hat{\boldsymbol{\sigma}}_{\mathbf{r}}-\operatorname{Tr}\left[\bar{\rho}_{t} \hat{\boldsymbol{\sigma}}_{\mathbf{r}}\right]-\int d \mathbf{r}^{\prime}\left(\hat{\rho}_{\mathbf{r}^{\prime}}-\rho\left(\mathbf{r}^{\prime}, t\right)\right) \frac{\delta}{\delta \rho\left(\mathbf{r}^{\prime}, t\right)}\left[\operatorname{Tr}\left[\bar{\rho}_{t} \hat{\mathbf{K}}_{\mathbf{r}}\right]+\operatorname{Tr}\left[\bar{\rho}_{t} \hat{\boldsymbol{\Pi}}_{\mathbf{r}}\right]\right] \\
& -\int d \mathbf{r}^{\prime}\left(\hat{\mathbf{g}}_{\mathbf{r}^{\prime}}-\mathbf{g}\left(\mathbf{r}^{\prime}, t\right)\right) \frac{\delta}{\delta \mathbf{g}\left(\mathbf{r}^{\prime}, t\right)}\left[\operatorname{Tr}\left[\bar{\rho}_{t} \hat{\mathbf{K}}_{\mathbf{r}}\right]+\operatorname{Tr}\left[\bar{\rho}_{t} \hat{\boldsymbol{\Pi}}_{\mathbf{r}}\right]\right] \\
= & \hat{\boldsymbol{\sigma}}_{\mathbf{r}}-\operatorname{Tr}\left[\bar{\rho}_{t} \hat{\boldsymbol{\sigma}}_{\mathbf{r}}\right]-\int d \mathbf{r}^{\prime}\left(\hat{\rho}_{\mathbf{r}^{\prime}}-\rho\left(\mathbf{r}^{\prime}, t\right)\right) \frac{\delta}{\delta \rho\left(\mathbf{r}^{\prime}, t\right)}\left[\frac{k_{B} T}{m} \rho(\mathbf{r}) \boldsymbol{\delta}+\frac{\mathbf{g}(\mathbf{r}) \mathbf{g}(\mathbf{r})}{\rho(\mathbf{r})}+\operatorname{Tr}\left[\bar{\rho}_{t} \hat{\boldsymbol{\Pi}}_{\mathbf{r}}\right]\right] \\
& -\int d \mathbf{r}^{\prime}\left(\hat{\mathbf{g}}_{\mathbf{r}^{\prime}}-\mathbf{g}\left(\mathbf{r}^{\prime}, t\right)\right) \frac{\delta}{\delta \mathbf{g}\left(\mathbf{r}^{\prime}, t\right)}\left[\frac{k_{B} T}{m} \rho(\mathbf{r}) \boldsymbol{\delta}+\frac{\mathbf{g}(\mathbf{r}) \mathbf{g}(\mathbf{r})}{\rho(\mathbf{r})}+\operatorname{Tr}\left[\bar{\rho}_{t} \hat{\boldsymbol{\Pi}}_{\mathbf{r}}\right]\right]
\end{aligned}
$$

where we have decomposed the kinetic and virial parts of the stress tensor and used (64). The ideal part and the virial part are independent of momentum variables, the latter because the virial stress tensor $\hat{\boldsymbol{\Pi}}_{\mathbf{r}}$ does not depend on velocities of the particles. The only term that depends on momentum is the convective term. Therefore,

$$
\begin{aligned}
\mathcal{Q}_{t} \hat{\boldsymbol{\sigma}}_{\mathbf{r}}^{\alpha \beta}= & \hat{\boldsymbol{\sigma}}_{\mathbf{r}}^{\alpha \beta}-\operatorname{Tr}\left[\bar{\rho}_{t} \hat{\boldsymbol{\sigma}}_{\mathbf{r}}^{\alpha \beta}\right]-\int d \mathbf{r}^{\prime}\left(\hat{\rho}_{\mathbf{r}^{\prime}}-\rho\left(\mathbf{r}^{\prime}, t\right)\right) \frac{\delta}{\delta \rho\left(\mathbf{r}^{\prime}, t\right)}\left[\frac{k_{B} T}{m} \rho(\mathbf{r}) \delta^{\alpha \beta}+\frac{\mathbf{g}^{\alpha}(\mathbf{r}) \mathbf{g}^{\beta}(\mathbf{r})}{\rho(\mathbf{r})}+\operatorname{Tr}\left[\bar{\rho}_{t} \hat{\mathbf{\Pi}}_{\mathbf{r}}^{\alpha \beta}\right]\right] \\
& -\int d \mathbf{r}^{\prime}\left(\hat{\mathbf{g}}_{\mathbf{r}^{\prime}}^{\gamma}-\mathbf{g}^{\gamma}\left(\mathbf{r}^{\prime}, t\right)\right) \frac{\delta}{\delta \mathbf{g}^{\gamma}\left(\mathbf{r}^{\prime}, t\right)} \frac{\mathbf{g}^{\alpha}(\mathbf{r}) \mathbf{g}^{\beta}(\mathbf{r})}{\rho(\mathbf{r})} \\
= & \hat{\sigma}_{\mathbf{r}}^{\alpha \beta}-\operatorname{Tr}\left[\bar{\rho}_{t} \hat{\boldsymbol{\sigma}}_{\mathbf{r}}^{\alpha \beta}\right]-\int d \mathbf{r}^{\prime}\left(\hat{\rho}_{\mathbf{r}^{\prime}}-\rho\left(\mathbf{r}^{\prime}, t\right)\right) \delta\left(\mathbf{r}-\mathbf{r}^{\prime}\right)\left[\frac{k_{B} T}{m} \delta^{\alpha \beta}-\mathbf{v}^{\alpha}(\mathbf{r}) \mathbf{v}^{\beta}(\mathbf{r})\right] \\
& -\int d \mathbf{r}^{\prime}\left(\hat{\rho}_{\mathbf{r}^{\prime}}-\rho\left(\mathbf{r}^{\prime}, t\right)\right) \frac{\delta}{\delta \rho\left(\mathbf{r}^{\prime}, t\right)} \operatorname{Tr}\left[\bar{\rho}_{t} \hat{\mathbf{\Pi}}_{\mathbf{r}}^{\alpha \beta}\right]-\int d \mathbf{r}^{\prime}\left(\hat{\mathbf{g}}_{\mathbf{r}^{\prime}}^{\gamma}-\mathbf{g}^{\gamma}\left(\mathbf{r}^{\prime}, t\right)\right) \delta\left(\mathbf{r}-\mathbf{r}^{\prime}\right)\left[\delta^{\gamma \beta} \mathbf{v}^{\alpha}(\mathbf{r})+\delta^{\alpha \gamma} \mathbf{v}^{\beta}(\mathbf{r})\right]
\end{aligned}
$$


Simplifying

$$
\begin{aligned}
\mathcal{Q}_{t} \hat{\boldsymbol{\sigma}}_{\mathbf{r}}^{\alpha \beta}= & \hat{\boldsymbol{\sigma}}_{\mathbf{r}}^{\alpha \beta}-\operatorname{Tr}\left[\bar{\rho}_{t} \hat{\boldsymbol{\sigma}}_{\mathbf{r}}^{\alpha \beta}\right]-\left(\hat{\mathbf{g}}_{\mathbf{r}}^{\beta}-\mathbf{g}^{\beta}(\mathbf{r}, t)\right) \mathbf{v}^{\alpha}(\mathbf{r})+\left(\hat{\mathbf{g}}_{\mathbf{r}}^{\alpha}-\mathbf{g}^{\alpha}(\mathbf{r}, t)\right) \mathbf{v}^{\beta}(\mathbf{r}) \\
& -\int d \mathbf{r}^{\prime}\left(\hat{\rho}_{\mathbf{r}^{\prime}}-\rho\left(\mathbf{r}^{\prime}, t\right)\right) \frac{\delta}{\delta \rho\left(\mathbf{r}^{\prime}, t\right)} \operatorname{Tr}\left[\bar{\rho}_{t} \hat{\mathbf{\Pi}}_{\mathbf{r}}^{\alpha \beta}\right]-\left(\hat{\rho}_{\mathbf{r}^{\prime}}-\rho\left(\mathbf{r}^{\prime}, t\right)\right) \frac{k_{B} T}{m} \delta^{\alpha \beta} .
\end{aligned}
$$

We may use now the same argument as in the case of the projected force for computing the last functional derivative. The final result for the projected currents is

$$
\begin{aligned}
\mathcal{Q}_{t} \hat{\mathbf{F}}_{\mathbf{r}}^{s \rightarrow 1}= & \hat{\mathbf{F}}_{\mathbf{r}}^{s \rightarrow 1}-\operatorname{Tr}\left[\bar{\rho}_{t} \hat{\mathbf{F}}_{\mathbf{r}}^{\mathrm{s} \rightarrow 1}\right]-\int d \mathbf{r}^{\prime} \int d \mathbf{r}^{\prime \prime}\left(\hat{\rho}_{\mathbf{r}^{\prime}}-\rho\left(\mathbf{r}^{\prime}, t\right)\right)\left\langle\delta \hat{\rho}_{\mathbf{r}^{\prime}} \delta \hat{\rho}_{\mathbf{r}^{\prime \prime}}\right\rangle^{-1}\left\langle\delta \hat{\rho}_{\mathbf{r}^{\prime \prime}} \hat{\mathbf{F}}_{\mathbf{r}}^{s \rightarrow 1}\right\rangle, \\
\mathcal{Q}_{t} \hat{\sigma}_{\mathbf{r}}^{\alpha \beta}= & \hat{\sigma}_{\mathbf{r}}^{\alpha \beta}-\operatorname{Tr}\left[\bar{\rho}_{t} \hat{\boldsymbol{\sigma}}_{\mathbf{r}}^{\alpha \beta}\right]-\left(\hat{\mathbf{g}}_{\mathbf{r}}^{\beta}-\mathbf{g}^{\beta}(\mathbf{r}, t)\right) \mathbf{v}^{\alpha}(\mathbf{r})+\left(\hat{\mathbf{g}}_{\mathbf{r}}^{\alpha}-\mathbf{g}^{\alpha}(\mathbf{r}, t)\right) \mathbf{v}^{\beta}(\mathbf{r}) \\
& -\int d \mathbf{r}^{\prime} \int d \mathbf{r}^{\prime \prime}\left(\hat{\rho}_{\mathbf{r}^{\prime}}-\rho\left(\mathbf{r}^{\prime}, t\right)\right)\left\langle\delta \hat{\rho}_{\mathbf{r}^{\prime}} \delta \hat{\rho}_{\mathbf{r}^{\prime \prime}}\right\rangle^{-1}\left\langle\delta \hat{\rho}_{\mathbf{r}^{\prime \prime}} \hat{\mathbf{\Pi}}_{\mathbf{r}}^{\alpha \beta}\right\rangle-\left(\hat{\rho}_{\mathbf{r}^{\prime}}-\rho\left(\mathbf{r}^{\prime}, t\right)\right) \frac{k_{B} T}{m} \delta^{\alpha \beta} .
\end{aligned}
$$

Under the approximation that the system is close to equilibrium, the relevant ensemble is very similar to the equilibrium ensemble, and we may take $\mathbf{v}(\mathbf{r}, t) \simeq 0$ and $\rho(\mathbf{r}, t) \simeq \rho^{\mathrm{eq}}(\mathbf{r})$. This gives the final result for the projected currents

$$
\begin{aligned}
& \mathcal{Q}_{t} \hat{\mathbf{F}}_{\mathbf{r}}^{\mathrm{s} \rightarrow 1}=\hat{\mathbf{F}}_{\mathbf{r}}^{\mathrm{s} \rightarrow 1}-\operatorname{Tr}\left[\rho_{\mathrm{eq}} \hat{\mathbf{F}}_{\mathbf{r}}^{\mathrm{s} \rightarrow 1}\right]-\int d \mathbf{r}^{\prime} \int d \mathbf{r}^{\prime \prime}\left(\hat{\rho}_{\mathbf{r}^{\prime}}-\rho_{\mathrm{eq}}\left(\mathbf{r}^{\prime}\right)\right)\left\langle\delta \hat{\rho}_{\mathbf{r}^{\prime}} \delta \hat{\rho}_{\mathbf{r}^{\prime \prime}}\right\rangle_{\mathrm{eq}}^{-1}\left\langle\delta \hat{\rho}_{\mathbf{r}^{\prime \prime}} \hat{\mathbf{F}}_{\mathbf{r}}^{\mathrm{s} \rightarrow 1}\right\rangle_{\mathrm{eq}}, \\
& \mathcal{Q}_{t} \hat{\boldsymbol{\sigma}}_{\mathbf{r}}^{\alpha \beta}=\hat{\boldsymbol{\sigma}}_{\mathbf{r}}^{\alpha \beta}-\operatorname{Tr}\left[\bar{\rho}_{t} \hat{\boldsymbol{\sigma}}_{\mathbf{r}}^{\alpha \beta}\right]-\int d \mathbf{r}^{\prime} \int d \mathbf{r}^{\prime \prime}\left(\hat{\rho}_{\mathbf{r}^{\prime}}-\rho_{\mathrm{eq}}\left(\mathbf{r}^{\prime}\right)\right)\left\langle\delta \hat{\rho}_{\mathbf{r}^{\prime}} \delta \hat{\rho}_{\mathbf{r}^{\prime \prime}}\right\rangle^{-1}\left\langle\delta \hat{\rho}_{\mathbf{r}^{\prime}} \hat{\boldsymbol{\sigma}}_{\mathbf{r}}^{\alpha \beta}\right\rangle_{\mathrm{eq}} .
\end{aligned}
$$

Physically, the last integral terms are responsible to subtract from the equilibrium fluctuations of the force density and stress tensor that part that may still have a systematic dependence on the fluctuations of the density.

${ }^{1}$ L. Bocquet and E. Charlaix, Chem. Soc. Rev. 39, 1073 (2010).

${ }^{2}$ E. Lauga, M. P. Brenner, and H. A. Stone, in Handbook of Experimental Fluid Mechanics, edited by C. Tropea, A. Yarin, and J. F. Foss (Springer, 2007), Chap. 19.

${ }^{3}$ L. Bocquet and E. Lauga, Nat. Mater. 10, 334 (2011).

${ }^{4}$ R. Evans, Adv. Phys. 28, 143 (1979).

${ }^{5}$ H. Löwen, J. Phys.: Condens. Matter 15, V1 (2003).

${ }^{6}$ R. Evans, M. Oettel, R. Roth, and G. Kahl, J. Phys.: Condens. Matter 28, 240401 (2016).

${ }^{7}$ U. Marconi and P. Tarazona, J. Phys.: Condens. Matter 12, A413 (2000).

${ }^{8}$ Z. Guo, T. S. Zhao, and Y. Shi, Phys. Fluids 18, 067107 (2006).

${ }^{9}$ P. Español and H. Löwen, J. Chem. Phys. 131, 244101 (2009).

${ }^{10}$ M. Schmidt and J. M. Brader, J. Chem. Phys. 138, 214101 (2013).

${ }^{11}$ B. Goddard, A. Nold, N. Savva, G. Pavliotis, and S. Kalliadasis, Phys. Rev. Lett. 109, 120603 (2012).

${ }^{12}$ D. Bedeaux, A. Albano, and P. Mazur, Phys. A 82, 438 (1976).

${ }^{13}$ L. Bocquet and J.-L. Barrat, Phys. Rev. E 49, 3079 (1994).

${ }^{14}$ J. G. Anero, P. Español, and P. Tarazona, J. Chem. Phys. 139, 034106 (2013).

${ }^{15}$ M. Schmidt, Phys. Rev E 84, 051203 (2011).

${ }^{16}$ R. Wittkowski, H. Löwen, and H. H. R. Brand, J. Chem. Phys. 137, 224904 (2012).

${ }^{17}$ J. S. Hansen, P. J. Daivis, and B. D. Todd, Phys. Rev. E 80, 046322 (2009).

${ }^{18}$ J. Hansen, J. Dyre, P. Daivis, B. Todd, and H. Bruus, Phys. Rev. E 84, 036311 (2011).

${ }^{19}$ R. Piccirelli, Phys. Rev. 175, 77 (1968).

${ }^{20}$ B. Robertson, Phys. Rev. 144, 151 (1966).
${ }^{21}$ H. Grabert, Projection Operator Techniques in Nonequilibrium Statistical Mechanics (Springer, 1982).

${ }^{22}$ S. Grossmann, Z. Phys. 233, 74 (1970).

${ }^{23}$ J. Zhang, B. D. Todd, and K. P. Travis, J. Chem. Phys. 121, 10778 (2004).

${ }^{24}$ J. Hansen, P. Daivis, K. Travis, and B. Todd, Phys. Rev. E 76, 1 (2007).

${ }^{25}$ B. Todd, J. Hansen, and P. Daivis, Phys. Rev. Lett. 100, 195901 (2008).

${ }^{26}$ J. Hansen, B. D. Todd, and P. J. Daivis, Phys. Rev. E 84, 016313 (2011).

${ }^{27}$ D. Bedeaux and A. Albano, Phys. A 88, 574 (1977).

${ }^{28}$ J.-L. Barrat and F. Chiaruttini, Mol. Phys. 101, 1605 (2003).

${ }^{29}$ J. Petravic and P. Harrowell, J. Chem. Phys. 127, 174706 (2007).

${ }^{30}$ S. K. Kannam, B. D. Todd, J. S. Hansen, and P. J. Daivis, J. Chem. Phys. 135, 144701 (2011).

${ }^{31}$ K. Huang and I. Szlufarska, Phys. Rev. E 89, 032119 (2014).

${ }^{32}$ B. Ramos-Alvarado, S. Kumar, and G. P. Peterson, Phys. Rev. E 93, 023101 (2016).

${ }^{33}$ V. P. Sokhan, D. Nicholson, and N. Quirke, J. Chem. Phys. 117, 8531 (2002).

${ }^{34}$ R. I. Cukier, R. Kapral, J. R. Lebenhaft, and J. R. Mehaffey, J. Chem. Phys. 73, 5244 (1980).

${ }^{35}$ K. Kawasaki and J. Gunton, Phys. Rev. A 8, 2048 (1973).

${ }^{36}$ P. J. Cadusch, B. D. Todd, J. Zhang, and P. J. Daivis, J. Phys. A: Math. Theor. 41, 035501 (2008).

${ }^{37}$ H. B. Callen, Thermodynamics (John Wiley \& Sons, New York, 1960).

${ }^{38}$ P. Español, R. Delgado-Buscalioni, R. Everaers, R. Potestio, D. Donadio, and K. Kremer, J. Chem. Phys. 142, 064115 (2015).

${ }^{39}$ P. Schofield and J. R. Henderson, Proc. R. Soc., Ser. A 379, 231 (1982).

${ }^{40}$ I. Bitsanis, J. J. Magda, M. Tirrell, and H. T. Davis, J. Chem. Phys. 87, 1733 (1987).

${ }^{41}$ I. Bitsanis, S. A. Somers, H. T. Davis, and M. Tirrell, J. Chem. Phys. 93, 3427 (1990).

${ }^{42}$ T. Qian, C. Qiu, and P. Sheng, J. Fluid Mech. 611, 333-364 (2008).

${ }^{43}$ H. C. Öttinger, J. Non-Newtonian Fluid Mech. 152, 66 (2008).

${ }^{44}$ L. Sagis, Rev. Mod. Phys. 83, 1367 (2011).

${ }^{45}$ P. Español and A. Donev, J. Chem. Phys. 143, 234104 (2015). 